\title{
Dynamic instability of MRE embedded soft cored sandwich beam with non-conductive skins
}

\author{
S.K. Dwivedy* and M. Srinivas \\ Department of Mechanical Engineering, Indian Institute of Technology Guwahati, Guwahati, India
}

Received 12 February 2009

Revised 29 May 2010

\begin{abstract}
In this work the governing temporal equations of motions with complex coefficients have been derived for a three-layered unsymmetric sandwich beam with nonconductive skins and magnetorheological elastomer (MRE) embedded soft-viscoelastic core subjected to periodic axial loads using higher order sandwich beam theory, extended Hamilton's principle, and generalized Galerkin's method. The parametric instability regions for principal parametric and combination parametric resonances for first three modes have been determined for various end conditions with different shear modulus, core loss factors, number of MRE patches and different skin thickness. This work will find application in the design and application of sandwich structures for active and passive vibration control using soft core and MRE patches.
\end{abstract}

Keywords: Sandwich beam, magnetorheological elastomer, parametric instability, soft core, vibration

\section{Introduction}

For light weight and suppression of vibration sandwich beams are very popular in aeronautical, space, automobile, structural and under-water applications. Also, multilayered sandwich beam like structures can be used as vehicle appendages, robot arms and in many other industrial structures and equipments. Now days a wide range of skin and core materials depending on requirements and applications are available. In these structures, due to shearing of the viscoelastic cores in the sandwich structures the vibratory energy gets dissipated. In addition to the viscoelastic core, smart material like magnetorheological elastomer (MRE) can also be used as a core material whose rheological properties can be controlled rapidly and reversibly by the application of an external magnetic field. Here, the stiffness of the system can be actively changed by applying suitable magnetic field as the sandwich beams with MRE core possess field-controllable flexural rigidities due to the field-dependent shear modulus of the MRE core.

To make sandwich beam more light weight, foam type soft core have been used. To analyze, these types of soft cored sandwich beam, Frostig and Baruch [1], Frostig [2], Bozhevolnaya and Frostig [3], Frostig [4] and Frostig and Baruch [5] used a higher order theory in which the transverse deflection of the core layer was taken into account. Here, the transverse displacements of the top and bottom skin layers are different. Free vibration analyses have been carried out in these studies. It may be noted that prior to these studies, the core layers were assumed to be rigid in transverse direction and classical theories were used by considering same transverse displacement for the top and bottom skin layers. Librescu and Hause [6] showed that the transverse flexibility of the core affects the overall

\footnotetext{
*Corresponding author: Dr. S. K. Dwivedy, Professor, Mechanical Engineering Department, Indian Institute of Technology Guwahati, Guwahati 781039, India. Tel.: +91 361258 2670; Fax: +91 361 2690762; E-mail: dwivedy@iitg.ernet.in.
} 
behaviour such as stresses and displacements of the sandwich beams. They have used the higher order effects owing to the non-linearity of the displacement fields of the core caused by its flexibility in the vertical direction.

MRE was introduced by Shiga et al. [7] and Jolly et al. [8] and Carlson and Jolly [9] have shown the difference in the operating mechanism of MRE and magneto rheological fluid (MRF). They reported that MRE is much like MRF, except for the fact that chain-like structures of MRE are developed during the curing process, while the chain-like structures of MRF are formed during operation. Zhu [10] and Volkava [11] investigated that the field-dependent properties of MRE are more stable than those of MRF. Field dependent shear modulus of MRE is investigated by many researchers experimentally. Jolly et al. [8], Zhu [9], Volkava [10], Zhou and Zhang [12] and Ginder et al. [13] have investigated field dependent shear modulus of MRE experimentally by shearing MRE on material test systems. Demchuk and Kuzmin [14] analyzed experimentally the field dependent shear modulus by measuring the vibration of sandwich cantilever beams. Zhou and Wang [15] studied the dynamic property of a sandwich beam, composed of nonconductive skins and soft core with both MRE and non-MRE parts. Zhou and Wang [16] studied the dynamics of a sandwich beam with conductive skins and soft core with both MRE and non-MRE parts. Stepanov, et al. [17] studied the viscoelastic properties of MRE experimentally. In most of these cases, the equivalent stiffness was determined and the free vibration responses of the sandwich structures were studied using MRE based core materials.

In many cases, sandwich beams are subjected to periodic axial loads. In these systems, when the amplitude and frequency of the axial load exceeds certain critical values, the system starts to buckle and vibrates making the system unstable even much before the Euler buckling load. In such cases the system may behave as a parametrically excited system $[18,19]$.

Some researchers, for example Ray and Kar [20-22] considered parametrically excited sandwich beams and studied the dynamic instability of various types of sandwich structures subjected to periodic axial loads. In such cases, the core layer was assumed to be rigid and classical theory was used to develop the governing equation of motion. As same transverse displacement of the top and bottom layers are taken, in these analyses, one obtains a single equation of motion of Mathieu-Hill's type with complex coefficient. For soft cored sandwich beams, considering transverse flexibility in the core layer, Dwivedy et al. [23] used higher order theory to obtain the governing equation of motions of symmetric sandwich beams and determined the parametric instability regions of these systems for various boundary conditions. It has been shown that the use of classical theory in such cases lead to erroneous results. Dwivedy and Srinivas [24] determined the parametric instability of a clamped-pinned sandwich beam with a single MRE patch embedded in soft core and nonconductive skins. In this work a detailed study of the unsymmetric sandwich beam has not been made.

In most of the above cases three layered symmetric sandwich beam has been taken. But in actual applications, the top and bottom layers may not be of same thickness. Also, in many cases to prevent the vibration of the primary structure one may go for constraint layer damping treatment by adding a viscoelastic core and a metallic skin layer. Hence, in such cases, it is required to consider unsymmetric sandwich beams.

It is well known that when a conducting material moves in a magnetic field an emf is induced in the conductor and when a current carrying conductor placed in a magnetic field it experience a force. As a magnetic field is used to change the stiffness of the MRE patch, by using conductive skin layer, due to the above mentioned magnetomechanical interaction, the sandwich beam will be subjected to axial load and moment $[15,16]$. Hence, to avoid that one may use nonconductive skins for the sandwich beam.

From the above literature review on MRE embedded soft cored sandwich beam, it may be noted that study on MRE embedded soft cored unsymmetric sandwich beam with nonconductive skins subjected to parametric excitation has not been carried out extensively. Hence in the present work an attempt has been made to derive the governing equation of motion of such type of sandwich beam and determine the parametric instability regions to avoid vibration. Here, the top and bottom layers of the sandwich beam are composed of nonconductive material like alulight ${ }^{\circledR}[25]$. The core layer contains single or multiple patches of MRE embedded in the foam type viscoelastic core. Taking the ratio of the length to the thickness of the sandwich beam very large, the skins of the sandwich beams are modeled as Euler Bernoulli beams. Due to the use of foam like soft core materials higher order theory $[4,6,7,23]$ has been used in the modeling of the sandwich beam. With extensive laborious mathematical involvements, the governing equations of motions have been derived as given in the following sections. This work will find a wide range of applications in the design of foam and MRE core based sandwich structures with nonconductive skins. The developed instability plots may be used for effective control of vibration in the sandwich structures. 


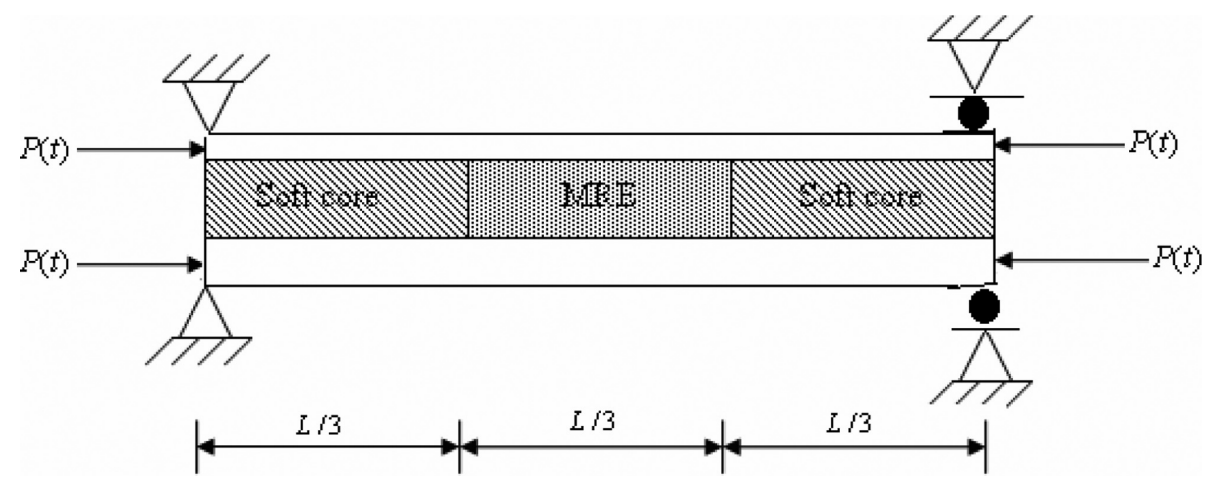

Fig. 1. Schematic diagram of an unsymmetric MRE embedded soft cored sandwich beam with simply supported end conditions subjected to periodic axial loads. (Dimensions not up to scale.).

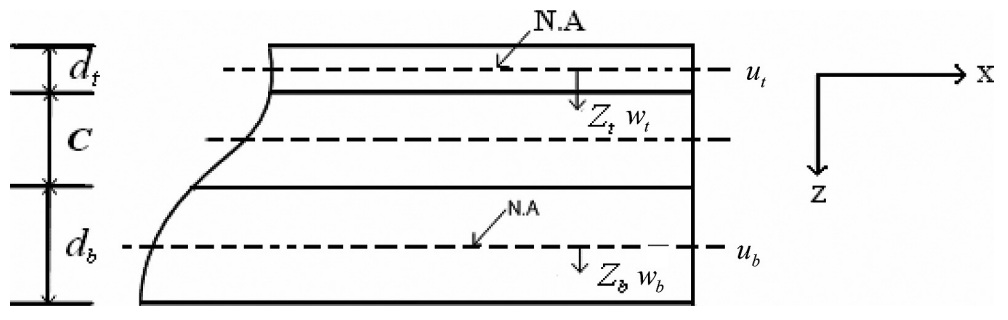

Fig. 2. Coordinate system and displacements of sandwich beam element.

\section{Modeling of sandwich beam}

Figure 1 shows a three layered simply supported unsymmetric, MRE embedded soft-cored sandwich beam with nonconductive skins. It is subjected to an axial periodic load $P(t)=P_{0}+P_{1} \cos \omega t$, where $\omega$ is the frequency of the applied load, $t$ is the time, $P_{0}$ and $P_{1}$ are the amplitude of static and dynamic loads, respectively. The load is acting on the whole cross-section of the skin layers of the sandwich beam at the ends. To simplify the analysis, it is shown by time varying point loads acting at the ends. The magnitude of the load is considered well below the critical Euler buckling load and the bending effect due to the unsymmetric loading is not considered. This loading is assumed to be similar to the loading considered in case of buckling of columns under static loading for various boundary conditions.

Figure 2 shows the coordinate system, neutral axis (NA), transverse displacements $w_{t}, w_{b}$; longitudinal displacements $u_{t}, u_{b}$ of the top and bottom skins which will be used in the derivation of the equations of motion.

The MRE part is configured with the chain-like structures embedded in the material perpendicular to the skins. The magnetic field is applied parallel to such chain-like structures i.e. it is perpendicular to the skins. Due to the use of nonconductive skins there will be no magnetoelatic loads acting on the skins during vibration (Zhou and Wang [17]). As a result, the bulk dynamic flexural rigidity of the sandwich beam can be controlled by the applied magnetic fields due to field-dependent shear modulus of the MRE part only.

The assumptions made for deriving the governing equations of motion are similar to that by Frostig and Baruch [3] and are as follows. The skins of the sandwich beam are modeled as Euler-Bernoulli beams and hence, the shear deformation and rotary inertia effects are neglected. This will hold well when the length to thickness of the sandwich beam is considered to be very large. The transversely flexible core layer is considered as a two dimensional elastic medium with small deformations where its height may change under loading, and its cross section does not remain planar. The longitudinal (in-plane) stresses in the core are neglected. The interface layers between the face sheets and the core are assumed to be infinitely rigid and provide perfect continuity of the deformations at the interfaces. There is no delamination between the layers and perfect bonding is assumed between layers of the sandwich beam.

The nomenclatures used in this analysis are given below. 


$\begin{array}{ll}A_{t}, A_{b}, A_{c} & \text { Area of cross section of top bottom and core layer respectively } \\ b & \text { Width of the beam } \\ B_{0} & \text { Magnetic field strength } \\ c & \text { Thickness of core layer } \\ d_{t}, d_{b} & \text { Thickness of top and bottom skin layers respectively } \\ E_{t}, E_{b}, E_{c} & \text { Young's modulus of elasticity for top, bottom and core layer respectively } \\ G_{c} & \text { Shear modulus of the core material } \\ G_{r} & \text { Shear modulus of the MRE material } \\ I_{t}, I_{b} & \text { Moment of Inertia of top and bottom skin with respect to their own neutral axis } \\ L & \text { Length of the beam } \\ m_{t}, m_{b}, m_{c} & \text { Mass per unit length of the top, bottom and core layer respectively } \\ m_{r} & \text { Mass of MRE layer per unit length } \\ \rho_{c} & \text { Density of the core layer } \\ u_{t}, u_{b} & \text { Longitudinal displacement of top and bottom skins (along X axis) } \\ w_{t}, w_{b} & \text { Transverse displacement of the top and bottom skin (along Z axis) } \\ (.) & \text { Derivative with respect to time } \\ ()_{x} & \text { Derivative with respect to } x \\ ()^{\prime} & \text { Nondimensional parameter corresponding to ( ) }\end{array}$

\subsection{Derivation of the equation of motion}

The potential energy $(U)$ of the MRE embedded soft cored sandwich beam in terms of stresses $(\sigma)$ and strains $(\varepsilon)$ is given by $[5,23]$,

$$
U=\int_{v_{\text {top }}} \sigma_{x x} \varepsilon_{x x} d v+\int_{v_{\text {bot }}} \sigma_{x x} \varepsilon_{x x} d v+\int_{v_{\text {core }}} \tau_{c} \gamma_{c} d v+\int_{v_{\text {core }}} \sigma_{z z} \varepsilon_{z z} d v
$$

Here $\sigma_{x x}$ and $\varepsilon_{x x}$ are the longitudinal normal stresses and strains in the top and bottom skins, $\tau_{c}$ and $\gamma_{c}$ are the shear stresses and strains in the core, $\sigma_{z z}$ and $\varepsilon_{z z}$ are the vertical normal stresses and strains in the core, $v_{t o p}, v_{b o t}$ and $v_{\text {core }}$ are the volumes of the top, bottom and core layer, respectively. It may be noted that for MRE patches the rigidity modulus is a function of the applied magnetic field. Hence one may actively control the stiffness of the structure by varying the magnetic field. Also, as nonconductive skins are used, the magnetic field will not introduce any axial force and couple in the skins.

The kinetic energy $\left(T_{k}\right)$ of the system is,

$$
T_{k}=(1 / 2)\left[\int_{0}^{L} m_{t}\left(\dot{u}_{t}^{2}+\dot{w}_{t}^{2}\right) d x+\int_{0}^{L} m_{b}\left(\dot{u}_{b}^{2}+\dot{w}_{b}^{2}\right) d x+\int_{v_{\text {core }}} \rho_{c} \dot{u}_{c}^{2} d v+\int_{v_{\text {core }}} \rho_{c} \dot{w}_{c}^{2} d v\right],
$$

where $m_{t}$ and $m_{b}$ are the mass per unit length of the top and bottom skins respectively, $\dot{u}_{t}$ and $\dot{u}_{b}$ are the velocities of the top and bottom skins in the horizontal direction, $\dot{w}_{t}$ and $\dot{w}_{b}$ are the velocities of the top and bottom skins in the vertical directions respectively, $\dot{u}_{c}$ and $\dot{w}_{c}$ are the core velocities in the horizontal and vertical directions respectively, $\rho_{c}$ is the density of the core, and $x$ is the longitudinal co-ordinate of the beam.

Unlike in the classical theory, where the core is considered to be rigid, in the present case with foam like soft core, the displacement of the core along $x$ direction $u_{c}$ and along $z$ direction $w_{c}$ can be written in terms of the longitudinal and transverse displacement of the top and bottom skins as follows (Frostig and Baruch [5], Dwivedy et al. [23]).

$$
u_{c}=u_{b}+\left(d_{b} / 2\right) w_{b, x}+\left(u_{t}-\left(d_{t} / 2\right) w_{t, x}\right)(1-(z / c)), \quad w_{c}=\left(w_{b}-w_{t}\right)(z / c)+w_{t}
$$

Unlike the case of Dwivedy et al. [23], where only soft viscoelastic core was taken, here MRE patches are embedded in the viscoelastic core.

The non-conservative work using the axial load $P$ is, 


$$
W_{n c}=\frac{1}{2} \int_{0}^{L} P\left(w_{t, x}^{2}+w_{b, x}^{2}\right) d x
$$

To derive the governing equations of motions of the system, denoting $D_{1}=\left(E_{t} I_{t}+E_{b} I_{b}\right)$, the following nondimensional terms have been used in the analysis.

$$
\begin{aligned}
& \bar{P}_{0}=P_{0} L^{2} / D_{1} ; \quad \bar{P}_{1}=P_{1} L^{2} / D_{1} ; \quad \xi_{c}=G_{c}^{*} A_{c} L^{2} / D_{1} ; \quad \phi_{t}=E_{t} A_{t} L^{2} / D_{1} ; \\
& \phi_{b}=E_{b} A_{b} L^{2} / D_{1} ; \quad t_{0}=\left(m l^{4} / D_{1}\right)^{1 / 2} ; \quad \bar{t}=\left(t / t_{0}\right) ; \bar{x}=(x / L) ; \\
& \bar{u}_{t}=\left(u_{t} / L\right) ; \quad \bar{u}_{b}=\left(u_{b} / L\right) ; \quad \bar{w}_{t}=\left(w_{t} / L\right) ; \quad \bar{w}_{b}=\left(w_{b} / L\right) ; \quad \bar{\omega}=\omega t_{0}
\end{aligned}
$$

Here $\bar{\omega}$ is the non-dimensional forcing frequency and $\bar{w}_{t}, \bar{w}_{b}, \bar{u}_{t}$ and $\bar{u}_{b}$ are the vertical and horizontal displacements of the top and bottom skins respectively in non-dimensional form $\bar{P}_{0}$ and $\bar{P}_{1}$ are respectively the non-dimensional static and dynamic load amplitudes, $G_{c}^{*}=G_{c}\left(1+j \eta_{c}\right)$ is the complex shear modulus of the viscoelastic core, where $G_{c}$ is the shear modulus and $j=\sqrt{-1}$ and $\eta_{c}$ is the core loss factor. To account for the shear of the core layer a term $g_{c}^{*}=g_{c}\left(1+j \eta_{c}\right)$ is used in the analysis, where $g_{c}=\left(\frac{G_{c}}{2 D_{1}}\right)\left(\frac{h_{c}}{h_{t}}\right)\left(\frac{L}{h_{t}}\right)^{2}$. This parameter is known as the shear parameter and the subscript $c=v$ or $e$ depending upon viscoelastic or MRE core materials, respectively.

Using Eqs (1-4) and applying extended Hamilton's principle yields the following sets of equations of motion.

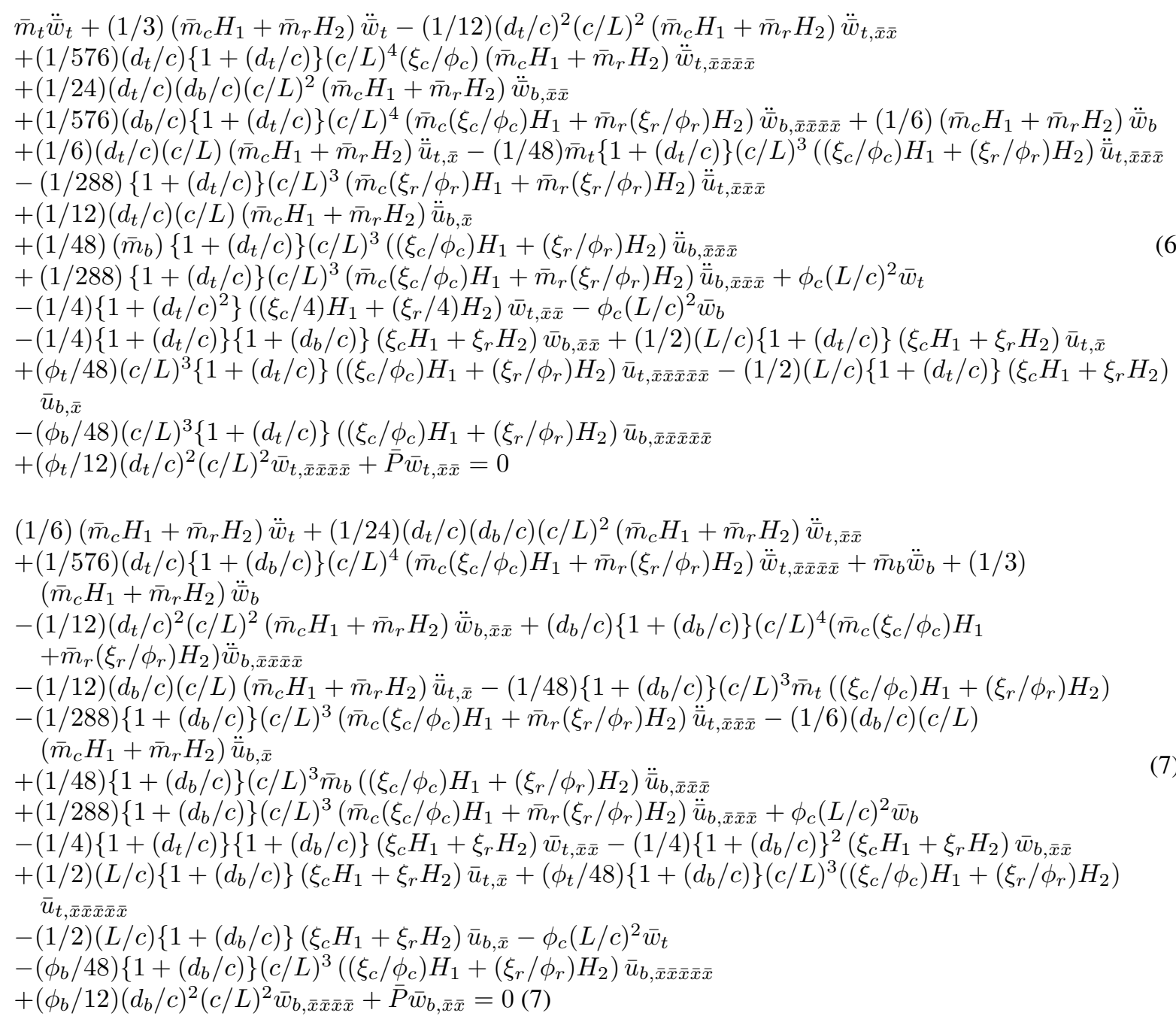




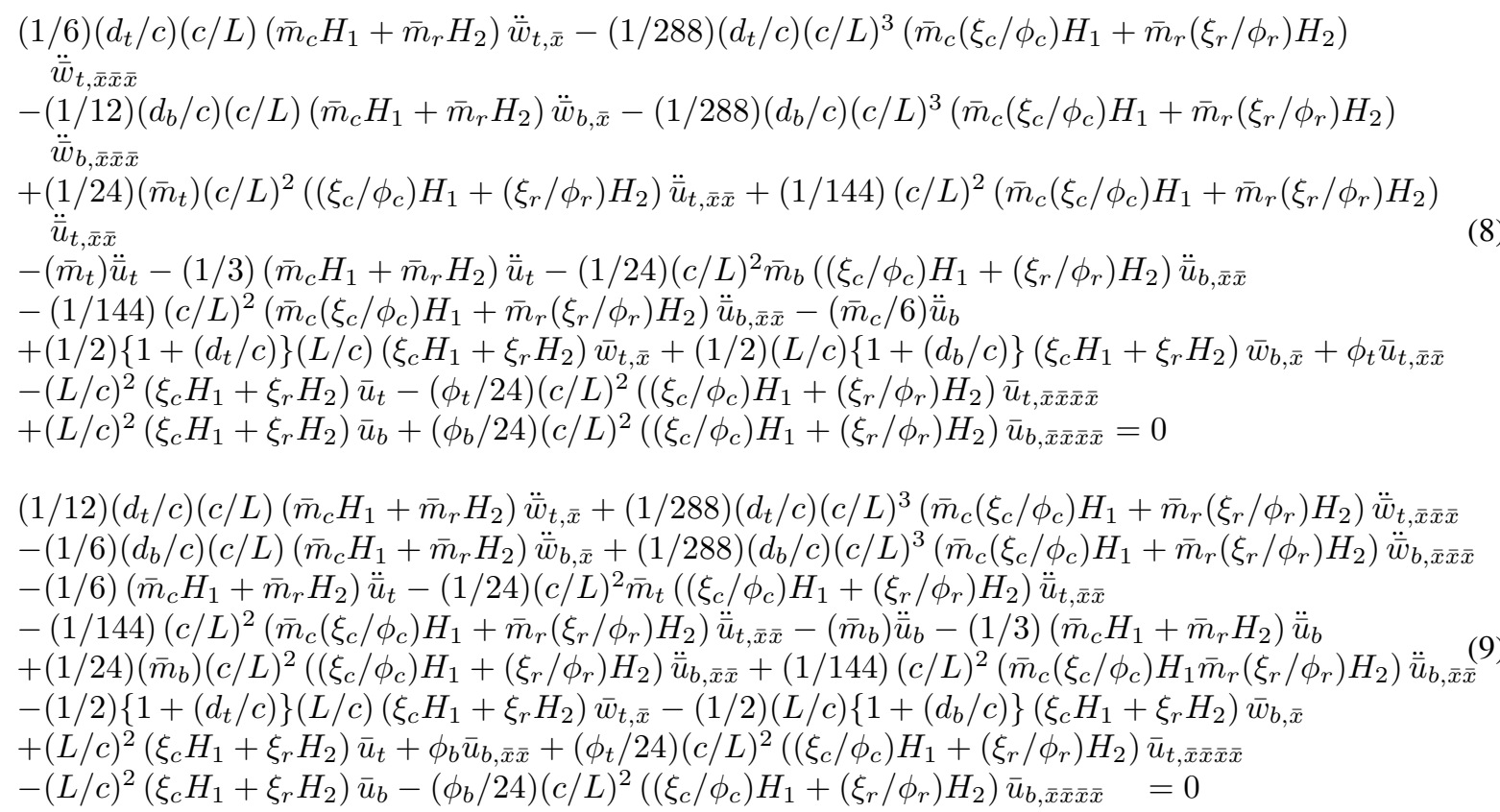

The locations of the MRE patches are included in the derivation by using the Heaviside function $H$. For example in case of the sandwich beam with MRE placed in the middle as shown in Fig. 1, in Eqs (6-9), Heaviside functions have been incorporated using $H_{1}$ and $H_{2}$, which are defined as follows.

$$
H_{1}=H(\bar{x})-H\left(\bar{x}-\bar{L}_{1}\right)+H\left(\bar{x}-\bar{L}_{2}\right) \text { and } H_{2}=H\left(\bar{x}-\bar{L}_{1}\right)-H\left(\bar{x}-\bar{L}_{2}\right),
$$

where $\bar{L}_{1}=1 / 3$ and $\bar{L}_{2}=2 / 3$.

For the sandwich beam with $n$ MRE patches placed alternatively with soft-core patch, following expressions for $H_{1}$ and $H_{2}$ should be taken.

$$
\begin{aligned}
& H_{1}=H(\bar{x})-H\left(\bar{x}-\bar{L}_{1}\right)+H\left(\bar{x}-\bar{L}_{2}\right)-H\left(\bar{x}-\bar{L}_{3}\right)+H\left(\bar{x}-\bar{L}_{4}\right)-\cdots+H\left(\bar{x}-\bar{L}_{n}\right), \\
& H_{2}=H\left(\bar{x}-\bar{L}_{1}\right)-H\left(\bar{x}-\bar{L}_{2}\right)+H\left(\bar{x}-\bar{L}_{3}\right)-H\left(\bar{x}-\bar{L}_{4}\right)+\cdots-H\left(\bar{x}-\bar{L}_{n}\right),
\end{aligned}
$$

where $\bar{L}_{1}=1 /(2 n+1), \bar{L}_{2}=2 /(2 n+1), \bar{L}_{3}=3 /(2 n+1), \cdots \bar{L}_{2 n}=2 n /(2 n+1)$.

\subsection{Approximate solution}

To obtain the approximate solutions for Eqs (6) to (9) one may write the transverse and longitudinal displacements of the systems by using the admissible functions $w_{m}(\bar{x}), w_{q}(\bar{x}), u_{r}(\bar{x}), u_{s}(\bar{x})$ corresponding to the location of the point at which the response is required and the time modulation as given below.

$$
\bar{w}_{t}=\sum_{m=1}^{N} w_{m}(\bar{x}) f_{m}(\bar{t}), \quad \bar{w}_{b}=\sum_{q=N+1}^{2 N} w_{q}(\bar{x}) f_{q}(\bar{t}), \quad \bar{u}_{t}=\sum_{r=2 N+1}^{3 N} u_{r}(\bar{x}) f_{r}(\bar{t}), \quad \bar{u}_{b}=\sum_{s=3 N+1}^{4 N} u_{s}(\bar{x}) f_{s}(\bar{t})
$$

where $N$ is the number of modes considered in the analysis, and $f_{m}(\bar{t}), f_{q}(\bar{t}), f_{r}(\bar{t})$, and $f_{s}(\bar{t})$ are the generalized co-ordinates and $w_{m}(\bar{x}), w_{q}(\bar{x}), u_{r}(\bar{x})$ and $u_{s}(\bar{x})$ are the shape functions chosen to satisfy as many of the boundary conditions as possible. Substituting Eq. (12) in Eqs (6-9) and applying generalized Galerkin's procedure one may obtain the following governing temporal equation of motion.

$$
[M]\{\ddot{f}\}+[K]\{f\}-\bar{P}_{1} \cos \bar{\omega} t[F]\{f\}=\{\phi\}
$$

Here

$$
\left(\dot{)}=d() / d \bar{t} \cdot\{f\}=\left\{\left\{f_{r}\right\}^{T}\left\{f_{m}\right\}^{T}\left\{f_{q}\right\}^{T}\left\{f_{s}\right\}^{T}\right\}^{T} .\right.
$$


The mass matrix $[M]$, stiffness matrix $[K]$, force vector $[F]$ have the same form as in the work of Dwivedy et al. [23]. But the elements of the sub matrices are different and are given in Appendix A. The used admissible functions are different for different boundary conditions and are given in Appendix B. It may be noted that Eq. (13) is a set of coupled equations and to solve these equations one may use modal analysis method to decouple these equations. Considering $[T]$ as the normalized modal matrix of $[M]^{-1}[K]$, using a new set of generalized coordinates $\{U\}$ and the linear transformation $\{f\}=[T]\{U\}$, one may write Eq. (13) in the following form.

$$
\ddot{U}_{q}+\left(\omega_{q}^{*}\right)^{2} U_{q}+2 \varepsilon \cos \bar{\omega} \bar{t} \sum_{p=1}^{4 N} b_{q p}^{*} U_{p}=0, \quad q=1 \ldots 4 N ;
$$

Here, $\varepsilon=\bar{P}_{1} / 2<1 .\left(\omega_{q}^{*}\right)^{2}$ represents the distinct $q^{\text {th }}$ eigenvalue of $[M]^{-1}[K]$ and $b_{q p}^{*}$ is the element of $[B]=$ $-[T]^{-1}[M]^{-1}[H][T]$. These two complex numbers can be written using their real and imaginary parts as $\omega_{q}^{*}=$ $\omega_{q, R}+j \omega_{q, I}$ and $b_{q p}^{*}=b_{q p, R}^{*}+j b_{q p, I}$. Here subscript $R$ stands for real and subscript $I$ stand for the imaginary part. In Eq. (14) as the coefficients of the response $\left(U_{p}\right)$ in the forcing term is a time varying parameter, these type of systems are known as parametrically excited system. Equation (14) is a set of coupled Mathieu-Hill's equations with complex coefficients. One may numerically solve these equations to obtain the response of the system.

As stated in Section 1, the parametrically excited systems undergo excessive vibration during principal parametric and combination parametric resonance conditions. Principal parametric resonance occurs when the excitation frequency of the system is nearly equal to twice the natural frequencies of the system and the combination parametric resonances occur when the excitation frequency of the system is nearly equal to the sum or difference of any two modal frequencies. It may be noted that corresponding to above mentioned frequencies, there exists critical values of amplitudes of excitations below which the system will not vibrate. Hence in this type of systems one may plot boundaries of instability regions to separate the stable and unstable state of the system. To obtain the boundaries of the regions of instability for simple (principal parametric) and combination parametric resonances following relations have been used (Ray and Kar [20]).

For simple or principal parametric resonance of $\mu^{\text {th }}$ mode

$$
\left|(\bar{\omega} / 2)-\omega_{\mu, R}\right|<\frac{1}{4}\left[\frac{4 \varepsilon^{2}\left(b_{\mu \mu, R}^{2}+b_{\mu \mu, I}^{2}\right)}{\omega_{\mu, R}^{2}}-16 \omega_{\mu, I}^{2}\right]^{1 / 2} .
$$

For combination resonance of sum type of $\mu^{t h}$ and $\nu^{\text {th }}$ modes

$$
\left|\bar{\omega}-\left(\omega_{\mu, R}+\omega_{v, R}\right)\right|<\frac{\left(\omega_{\mu, I}+\omega_{v, I}\right)}{4\left(\omega_{\mu, I} \omega_{v, I}\right)^{1 / 2}}\left[\frac{4 \varepsilon^{2}\left(b_{\mu v, R} b_{v \mu, R}+b_{\mu v, I} b_{v \mu, I}\right)}{\omega_{\mu, R} \omega_{v, R}}-16 \omega_{\mu, I} \omega_{v, I}\right]^{1 / 2} .
$$

For combination resonance of difference type of $\mu^{t h}$ and $\nu^{\text {th }}$ modes

$$
\left|\bar{\omega}-\left(\omega_{v, R}-\omega_{\mu, R}\right)\right|<\frac{\left(\omega_{\mu, I}+\omega_{v, I}\right)}{4\left(\omega_{\mu, I} \omega_{v, I}\right)^{1 / 2}}\left[\frac{4 \varepsilon^{2}\left(b_{\mu v, R} b_{v \mu, R}+b_{\mu v, I} b_{v \mu, I}\right)}{\omega_{\mu, R} \omega_{v, R}}-16 \omega_{\mu, I} \omega_{v, I}\right]^{1 / 2} .
$$

Numerically these regions have been determined for different system parameters for various boundary conditions as described in the following section.

\section{Results and discussions}

In this section the parametric instability region of the sandwich beams with MRE embedded viscoelastic core and alulight ${ }^{\circledR}[25]$ skins have been plotted for various boundary conditions viz., simply supported, clamped-guided and clamped-free boundary conditions. The effect of shear parameter, core loss factor, number of MRE patches and the skin thickness on the parametric instability regions of sandwich beam have been discussed. For this purpose three sandwich beams have been considered as shown in Figs 1, 3 and 4. In the first beam (Fig. 1) the core consists of two non -MRE and one MRE part. The core of the second beam (Fig. 3) is divided into five equal parts with soft-core 


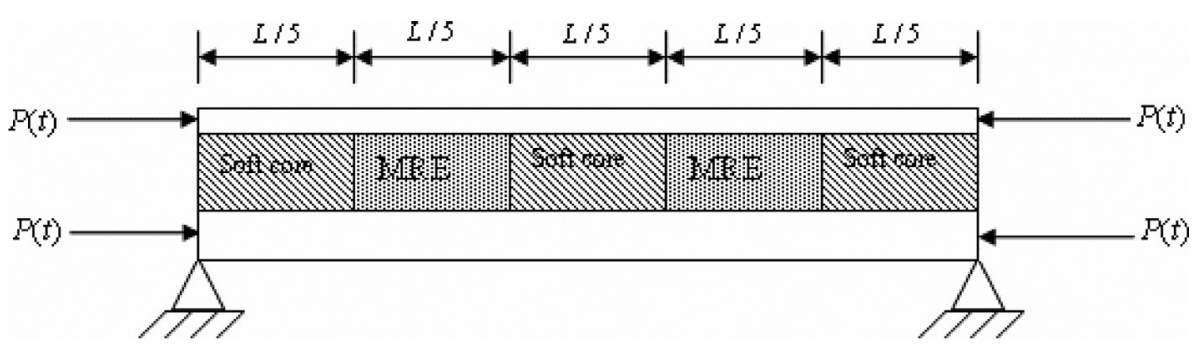

Fig. 3. Unsymmetric sandwich beam with alulight ${ }^{\circledR}$ skins showing relative position of two MRE patches embedded in the soft-core. (Dimensions not up to scale).

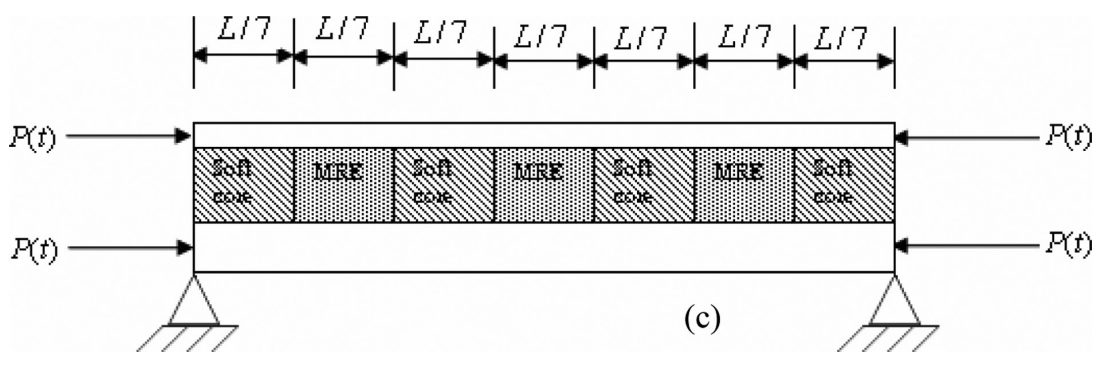

Fig. 4. Unsymmetric sandwich beam with alulight ${ }^{\circledR}$ skins showing relative position of three MRE patches embedded in the soft-core. (Dimensions not up to scale).

and MRE placed alternatively. The core of the third beam is divided into seven equal parts with soft-core and MRE placed alternately as shown in Fig. 4.

Following physical parameters are taken for the numerical analysis. The span of the beam, $L=230$ mm; width, $b=10 \mathrm{~mm}$; top face thickness $d_{t}=2 \mathrm{~mm}$ and bottom face thickness $d_{b}=10 \mathrm{~mm}$, core thickness, $c=4 \mathrm{~mm}$; Young's modulus of the alulight ${ }^{\circledR}$ skins, $E_{t}=E_{b}=5 \mathrm{GPa}[25] ; B_{0}=0.8$ Tesla; the zero-field shear modulus of MRE and the shear modulus of non-MRE soft-core are $0.388 \mathrm{MPa}$; the shear modulus of the MRE part at $B_{0}=0.8$ Tesla is 1.55 times that of its zero-field shear modulus [15], Young's modulus of the MRE part, which is assumed to be unchanged with the applied magnetic field and the non-MRE part are 1.7 MPa [15]; the densities of the alulight ${ }^{\circledR}$ skins [25] $\rho_{t}=\rho_{b}=500 \mathrm{~kg} / \mathrm{m}^{3}$; the density of the core, for both MRE part and the non-MRE part, is $1100 \mathrm{~kg} / \mathrm{m}^{3}$ [15], non dimensional static load $\bar{P}_{0}$ is taken to be 0.1 unless otherwise specified. The non dimensional frequency is given by, $\bar{\omega}=\omega t_{0}$ where $t_{0}=\left(m l^{4} / D_{1}\right)^{1 / 2}$.

In all the figures with instability regions, the ordinate $\bar{P}_{1}$ is the amplitude of non-dimensional dynamic load and the abscissa $\bar{\omega}$ is the non-dimensional forcing frequency. Also, in the parametric instability regions, the regions enclosed by the curves (e.g., marked $\mathrm{U}$ in Fig. 5) are unstable and the regions outside the curves (e.g., marked $\mathrm{S}$ in Fig. 5) are stable. In other words, if a system is operated with a frequency $\bar{\omega}$ and amplitude of excitation $\bar{P}_{1}$, and the corresponding point is inside the unstable region, the system response will grow with time and the system may fail due to excessive vibration. Similarly, if the operating condition is in the stable region, the system will be in the trivial state i.e., it will not vibrate. Hence, the designer and operator of the sandwich structure should have knowledge about the instability regions for safe operation of the system.

For visco-elastic materials, core loss factor $\left(\eta_{c}\right)$ is a measure of energy dissipation capacity and the shear parameter $\left(g_{c}\right)$ is a measure of stiffness of the material and is important in determining how much energy gets into the viscoelastic material. So these two parameters are varied in determining the instability regions for the parametrically excited beams. It may be noted that for the applied magnetic field of $0.8 \mathrm{~T}$, the shear parameter of the MRE part of the core is 1.55 times that of the non MRE part.

\subsection{Simply supported sandwich beam $(S-S)$}

Figure 5 shows the parametric instability regions for first three modes of an unsymmetric sandwich beam as shown in Fig. 1 with simply supported boundary conditions. This is obtained for the nondimensional static load $\bar{P}_{0}$ equal 

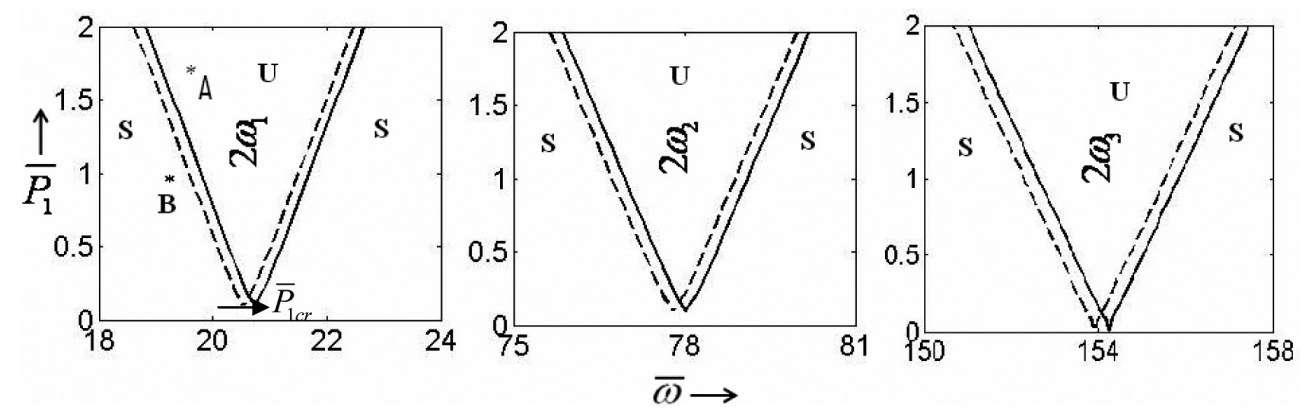

Fig. 5. Instability regions of S-S sandwich beam with alulight ${ }^{\circledR}$ skins and MRE placed in the middle of soft-core. $g=0.0163, \eta_{c}=0.1 .-$ $\bar{P}_{0}=0.1,---\bar{P}_{0}=0.2$.
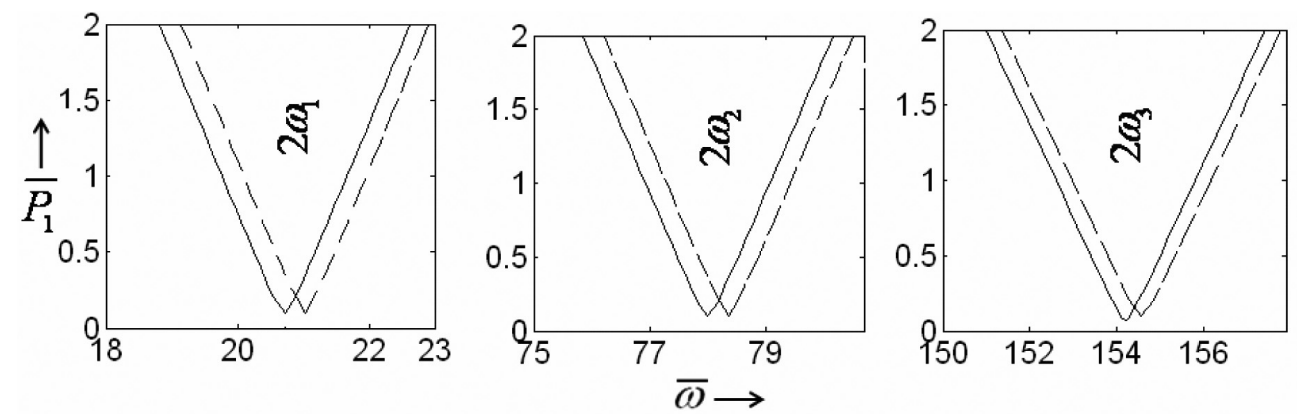

Fig. 6. Effect of shear parameter on instability regions for $\eta_{c}=0.1 .-g=0.0163,----g=0.021$.

to 0.1 and 0.2 . In both these cases the beam is found to be stable for combination resonances of sum and difference types. It may be noted from this figure that with decrease in $\bar{P}_{1}$ the unstable region decreases and the system can operate for a wide frequency range. Further, there exists a critical value of $\bar{P}_{1}\left(\bar{P}_{1 c r}\right)$ below which the system can operate at any frequency without vibration. For example, in this case $\bar{P}_{1 c r}$ equal to 0.1 for both first and second modes and 0.07 for the third mode.

It may be noted from Fig. 5, that with increase in $\bar{P}_{0}$, the instability region moves towards left and occurs at lower frequencies. This can be explained from the expression for the stiffness matrix $[K]$ given in appendix which shows that the stiffness of the system decreases with increase in $\bar{P}_{0}$. As the instability region occurs near twice the natural frequencies in case of principal parametric resonances, hence, the instability region occurs at lower excitation frequencies similar to the observations in case of symmetric sandwich beam [23].

The effect of shear parameter of the viscoelastic core $(g)$ on instability regions has been shown in Fig. 6. It is observed that with increase in shear parameter, while the range of parametric instability region remains unchanged, the region starts at a higher frequency. This is due to the fact that increase in shear parameter increases the effective stiffness of the system. In this case $\bar{P}_{1 c r}$ is equal to 0.1 for first two modes and 0.07 for third mode. For the third mode, for $g=0.021$, the critical value of $\bar{P}_{1 c r}$ is equal to 0.1 .

The effect of core loss factor $\eta_{c}$ on instability regions has been shown in Fig. 7. It is observed that with increase in core loss factor, the region of instability shifts upwards indicating the reduction of instability region. Hence the system will be more stable with increase in the core loss factor. It may be noted that with increase in $\eta_{c}$ from 0.1 to 0.6 , the critical amplitude of excitation $\bar{P}_{1 c r}$ increases from 0.2 to 0.6 for both first and second modes, and 0.07 to 0.4 for the third mode.

The effects of increase in the number of MRE patches have been studied by plotting the instability regions as shown in Fig. 8 for all the three beams shown in Figs 1, 3 and 4. One may note that in all the three modes, the instability regions shifts to higher values of frequencies with increase of MRE patches in the soft-core. This may be explained as below. As the shear parameter for the MRE part is taken 1.55 times more than that of the non MRE core for a magnetic field strength of 0.8 Tesla [17], with increase in the number of patches, the effective 

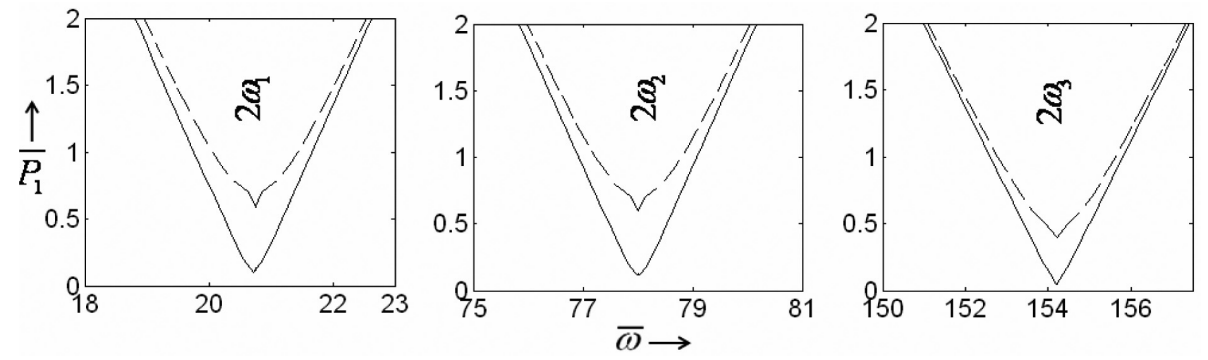

Fig. 7. Effect of core loss factor on instability regions for $g=0.0163$.
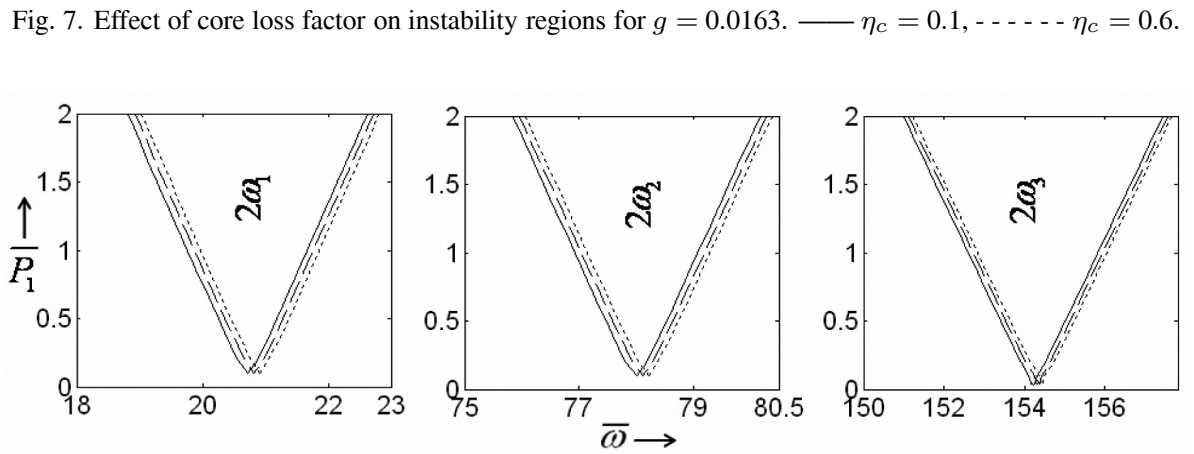

Fig. 8. Effect of number of MRE patches on parametric instability regions of the sandwich beam $\left(g=0.0163, \eta_{c}=0.1\right)$. One MRE patchTwo MRE patches - - - and Three MRE patches - - - - - .
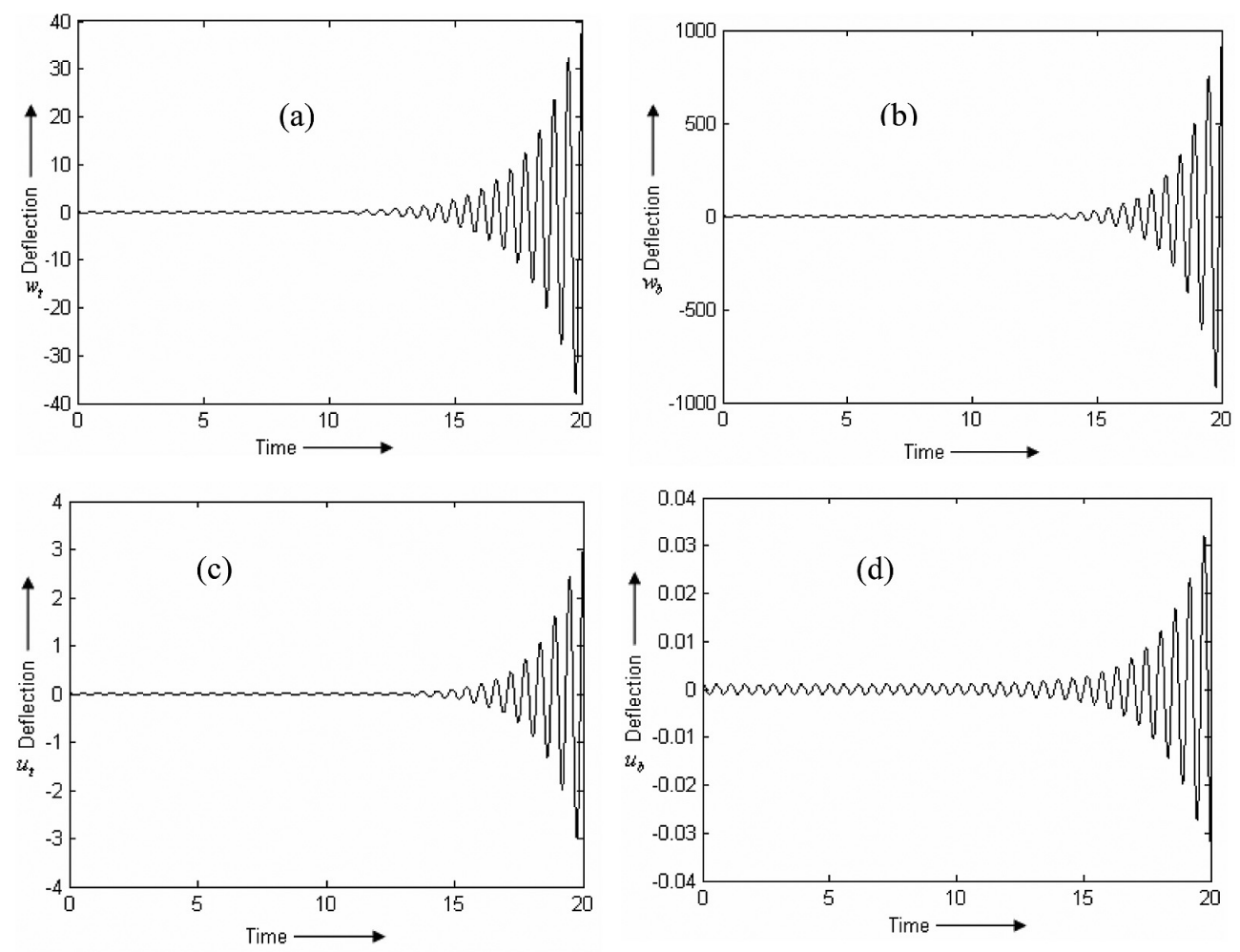

Fig. 9. Response of soft-cored sandwich beam with MRE (a) response of top skin in vertical direction ( $w_{t}$ ), (b) response of bottom skin in vertical direction $\left(w_{b}\right),(\mathrm{c})$ response of top skin in horizontal direction $\left(u_{t}\right),(\mathrm{d})$ response of bottom skin in horizontal direction $\left(u_{b}\right)$. 

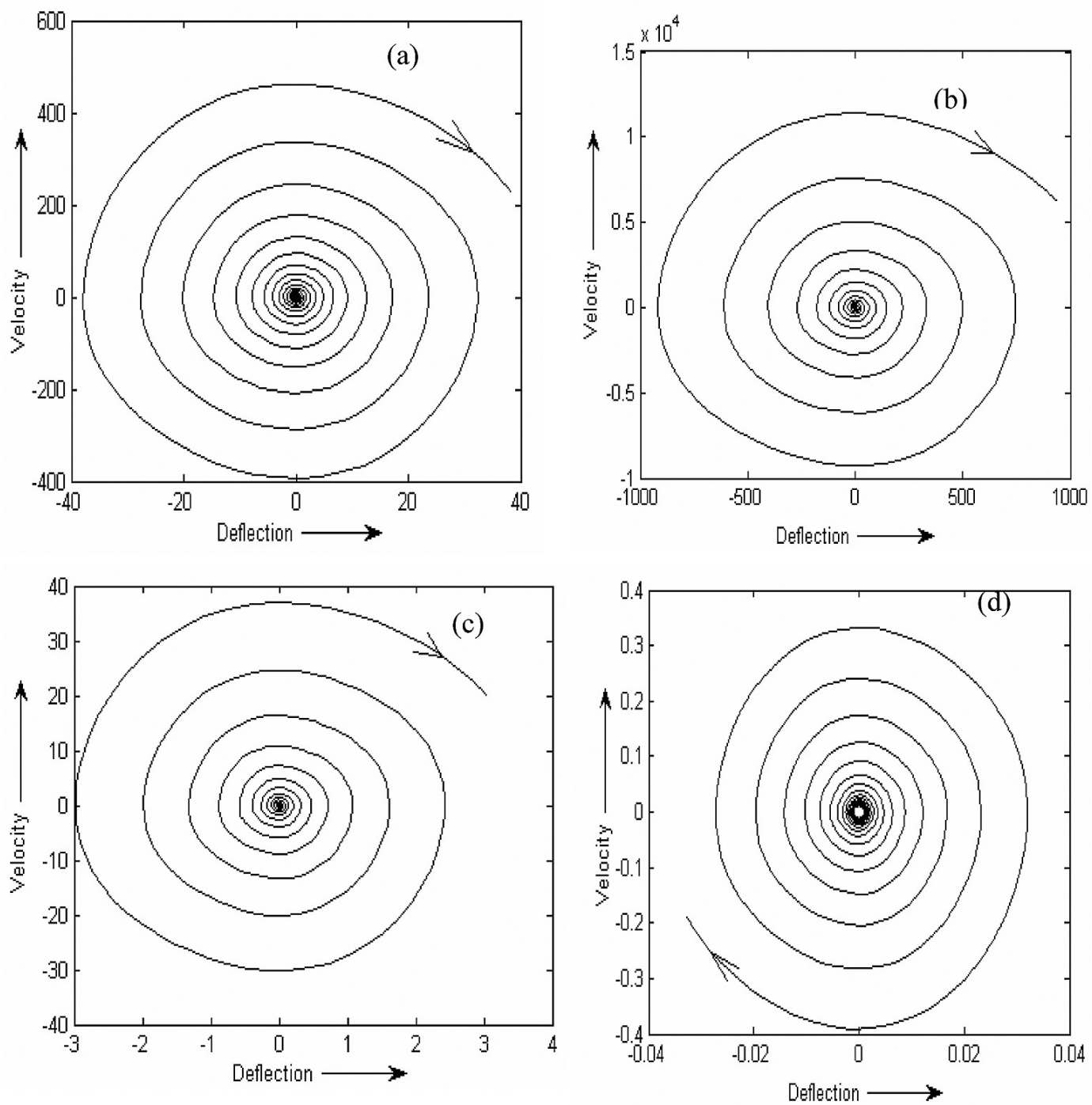

Fig. 10. Phase portrait of soft-cored sandwich beam with MRE (a) phase portrait of top skin in vertical direction, (b) phase portrait of bottom skin in vertical direction, (c) phase portrait of top skin in horizontal direction, (d) phase portrait of bottom skin in horizontal direction. Key corresponds to point A of Fig. 5 with $\bar{P}_{0}=0.1$.

stiffness of the sandwich beam increases and hence the natural frequency of the system increases. Since the principal parametric resonance condition occurs near twice the natural frequencies, hence with increase in the number of patches, the instability regions shifts to higher frequencies in all the three modes. The beam is found to be stable for the combination resonances of both sum and difference type for all the above cases. It may be noted that, these instability regions are plotted with shear parameter $g$ equal to 0.0163 and core loss factor $\eta_{c}=0.1$.

As the use of a number of patches only increase the stiffness of the system, hence, the natural or modal frequencies increases with increase in number of patches. This in turn shifts the instability regions towards right as they occur near twice the natural frequencies. Hence, from Figs 7 and 8, one may note that, use of a single MRE patch with higher core loss factor gives better stability, than using a number of MRE patch with lower core loss factor. So, in order to have a higher value of $\bar{P}_{1 c r}$ or a better stable system as in case of Fig. 7, one may suitably change the core loss factor actively by changing the magnetic field or passively by using MRE patch of higher core loss factor.

To validate the results obtained by using the expressions given in Eqs (15-17), one may determine the response and phase portrait by solving the temporal equation of motion numerically. Figure 9 shows the time response 

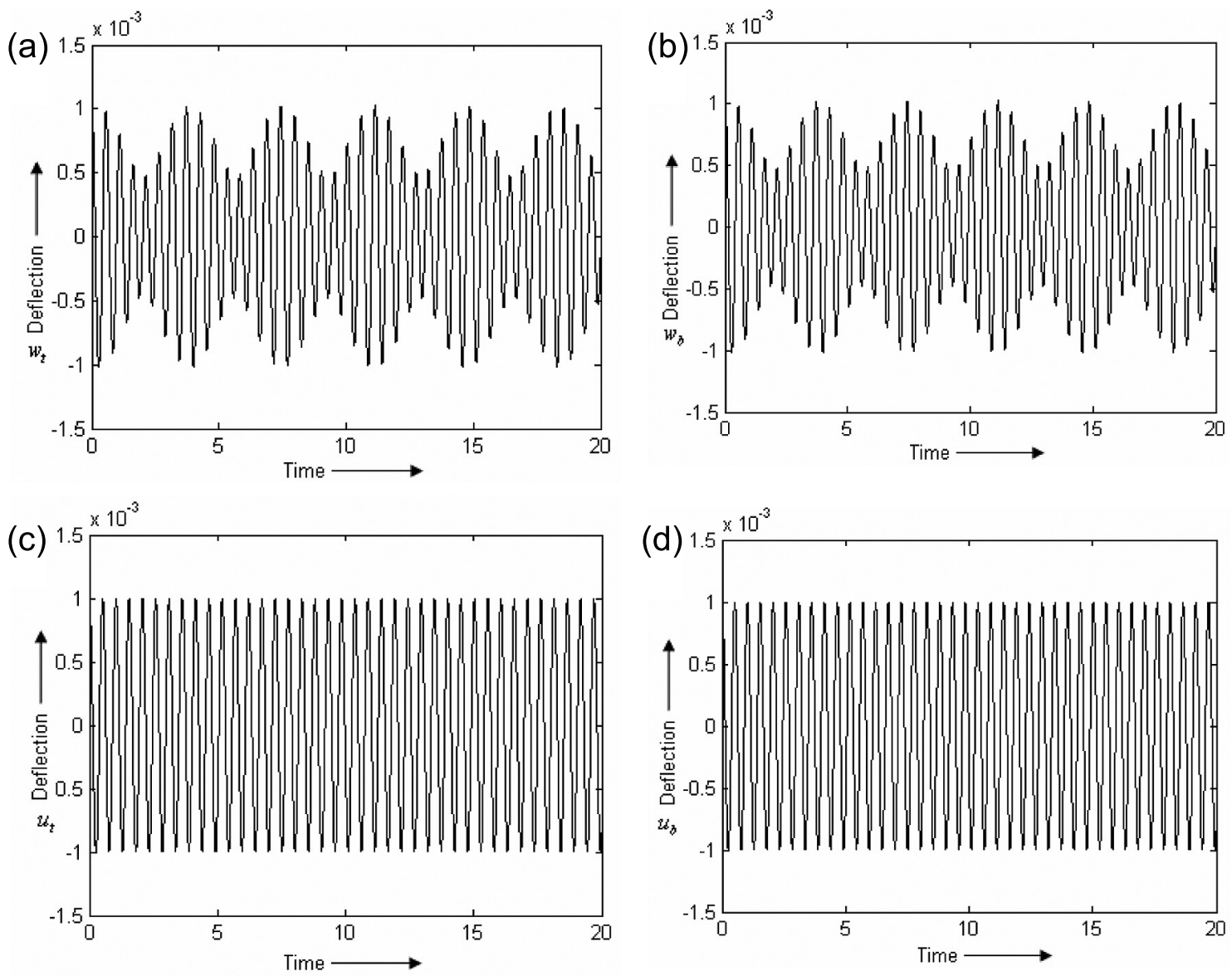

Fig. 11. Response of soft-cored sandwich beam with MRE (a) response of top skin in vertical direction ( $w_{t}$ ), (b) response of bottom skin in vertical direction $\left(w_{b}\right)$, (c) response of top skin in horizontal direction $\left(u_{t}\right)$, (d) response of bottom skin in horizontal direction $\left(u_{b}\right)$. Key correspond to point B of Fig. 5 with $\bar{P}_{0}=0.1$.

curves and Fig. 10 shows the corresponding phase portraits for both longitudinal and transverse displacements of the skins of the sandwich beam obtained by solving numerically Eq. (13) for the frequency and amplitude of excitation corresponding to point A with $\bar{P}_{0}=0.1$ as marked in Fig. 5.

It clearly shows the response to be unstable as predicted in Fig. 5. Similarly, Figures 11 and 12 show the response curves and phase portraits for both longitudinal and transverse displacements of the skins of the sandwich beam obtained by solving numerically Eq. (13) for the frequency and amplitude of excitation corresponding to point B with $\bar{P}_{0}=0.1$ (Fig. 5). The response is clearly observed to be bounded and stable which is same as observed in Fig. 5. Similar response curves and phase plots have been observed for other points in the instability regions in all the boundary conditions and hence they have not been plotted.

Keeping the top layer thickness $2 \mathrm{~mm}$ and other parameter same as in Fig. 5, the effect of variation of bottom layer thickness on instability regions is studied for a single MRE patch embedded sandwich beam as shown in Fig. 13. For the first mode, with decrease in bottom layer thickness from $10 \mathrm{~mm}$ to $2 \mathrm{~mm}$, as both stiffness and mass of the system decreases, hence, the instability regions shift towards left. It may be noted that the same reason is not valid for the second and third modes. In the second and third modes, with decrease in thickness of the bottom layer, the instability regions move towards right indicating an increase in modal frequencies. It may be due to the formation of a single node in case of second mode and two nodes in case of third mode, which increases the stiffness of the system. One may observe similar effect by changing the top layer thickness. It may further be noted that, change in skin thickness affects mass, stiffness and the forcing parameters and hence, for correct prediction of instability regions, one should use the developed equations to find them. 

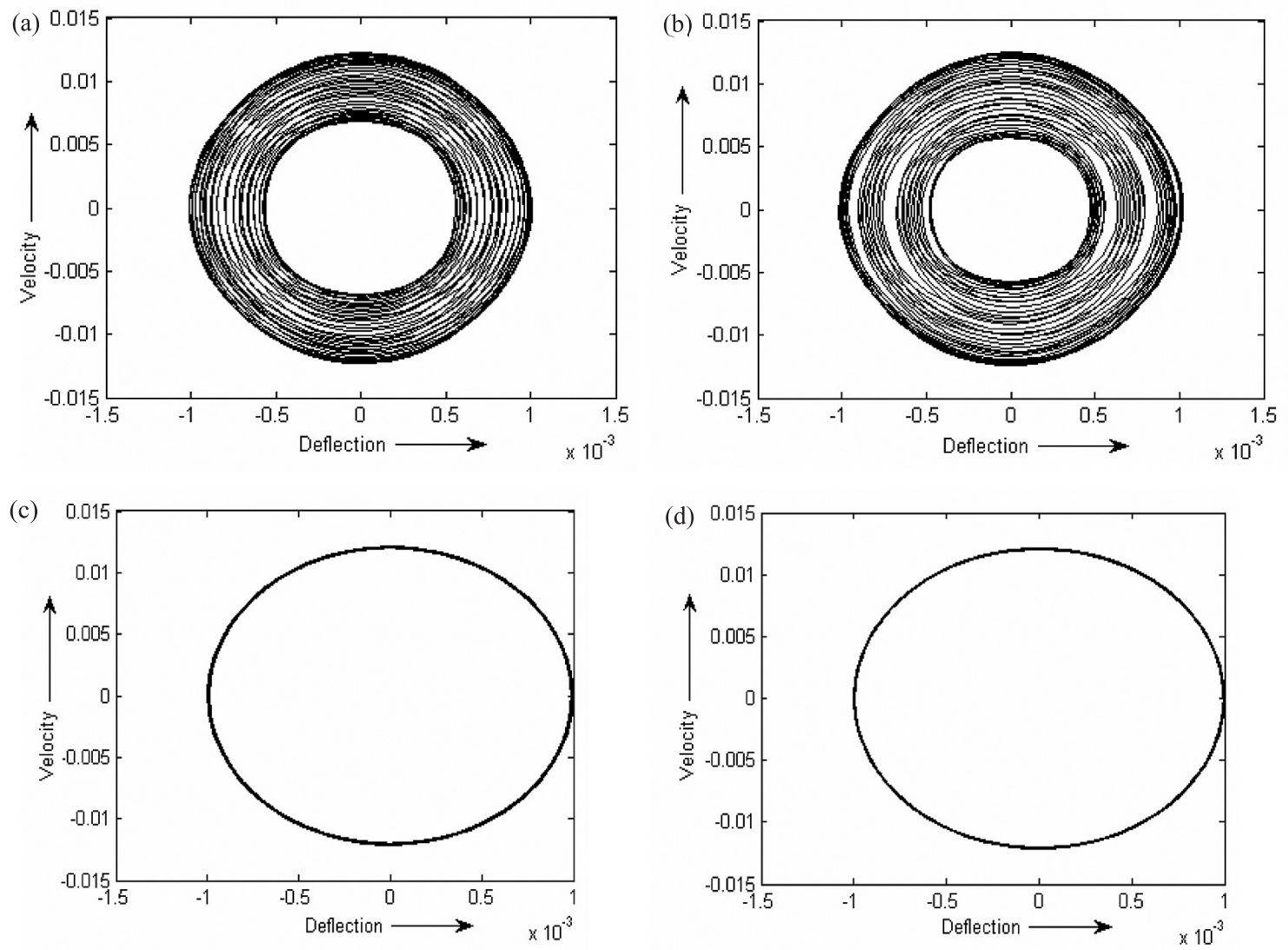

Fig. 12. Phase portrait of soft-cored sandwich beam with MRE (a) phase portrait of top skin in vertical direction, (b) phase portrait of bottom skin in vertical direction, (c) phase portrait of top skin in horizontal direction, (d) Phase portrait of bottom skin in horizontal direction. Key s correspond to point B of Fig. 5 with $\bar{P}_{0}=0.1$.
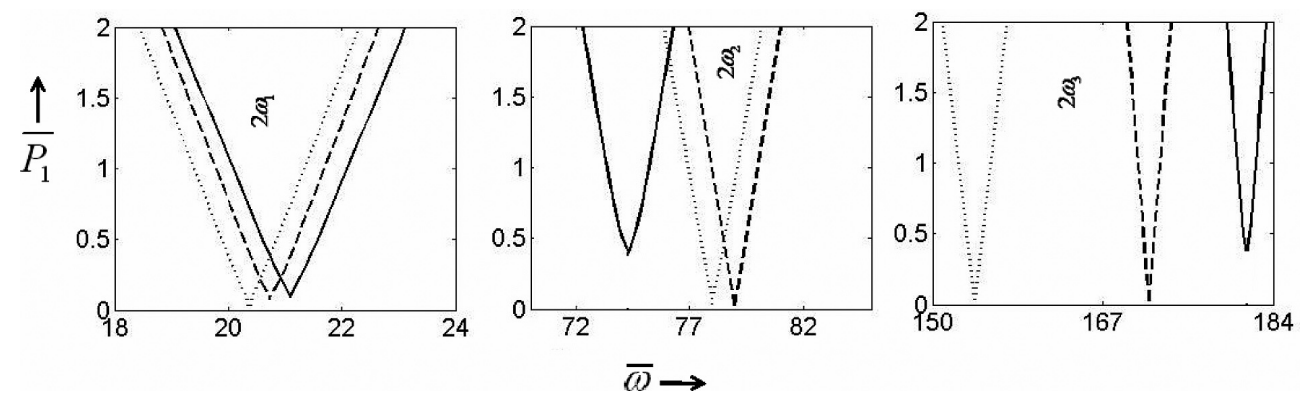

Fig. 13. Effect of variation of bottom skin thickness on instability regions. $-d_{b}=10 \mathrm{~mm},---d_{b}=6 \mathrm{~mm},----d_{b}=2 \mathrm{~mm}$. Other parameters same as in Fig. 5 with $\bar{P}_{0}=0.1$.

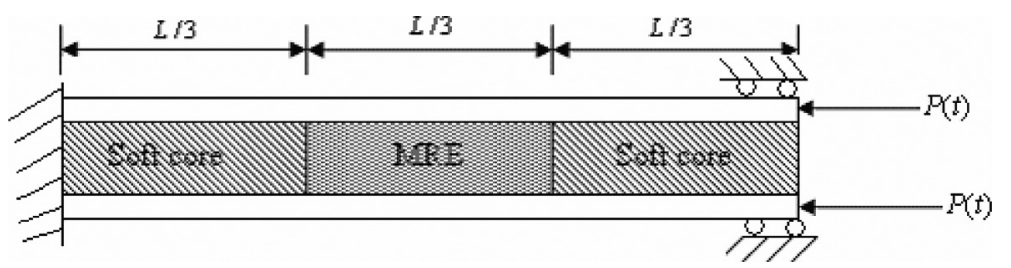

Fig. 14. Schematic diagram of MRE embedded soft cored sandwich beam with clamped -guided end conditions. (Dimensions not up to scale.). 

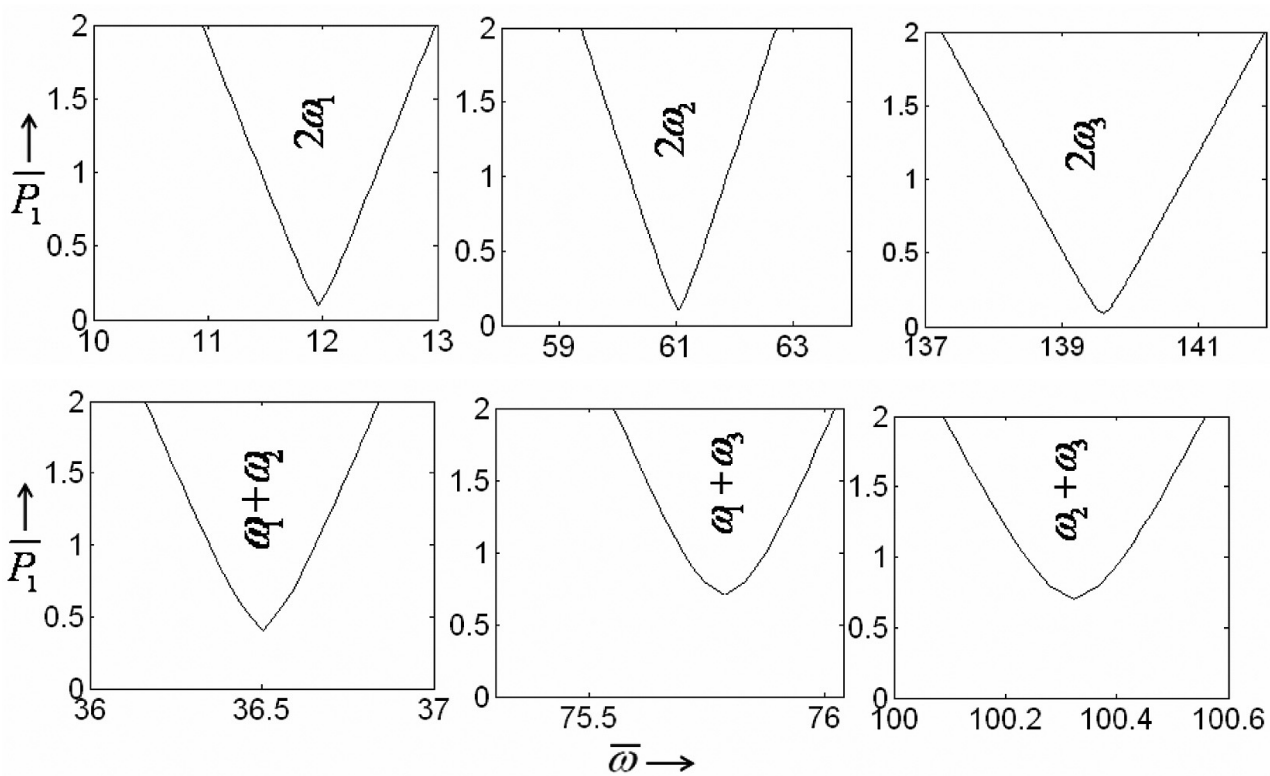

Fig. 15. Instability regions of $\mathrm{C}-\mathrm{G}$ sandwich beam with alulight ${ }^{\circledR}$ skins and MRE placed in the middle of soft-core. Key as in Fig. 5 with $\bar{P}_{0}=$ 0.1 .
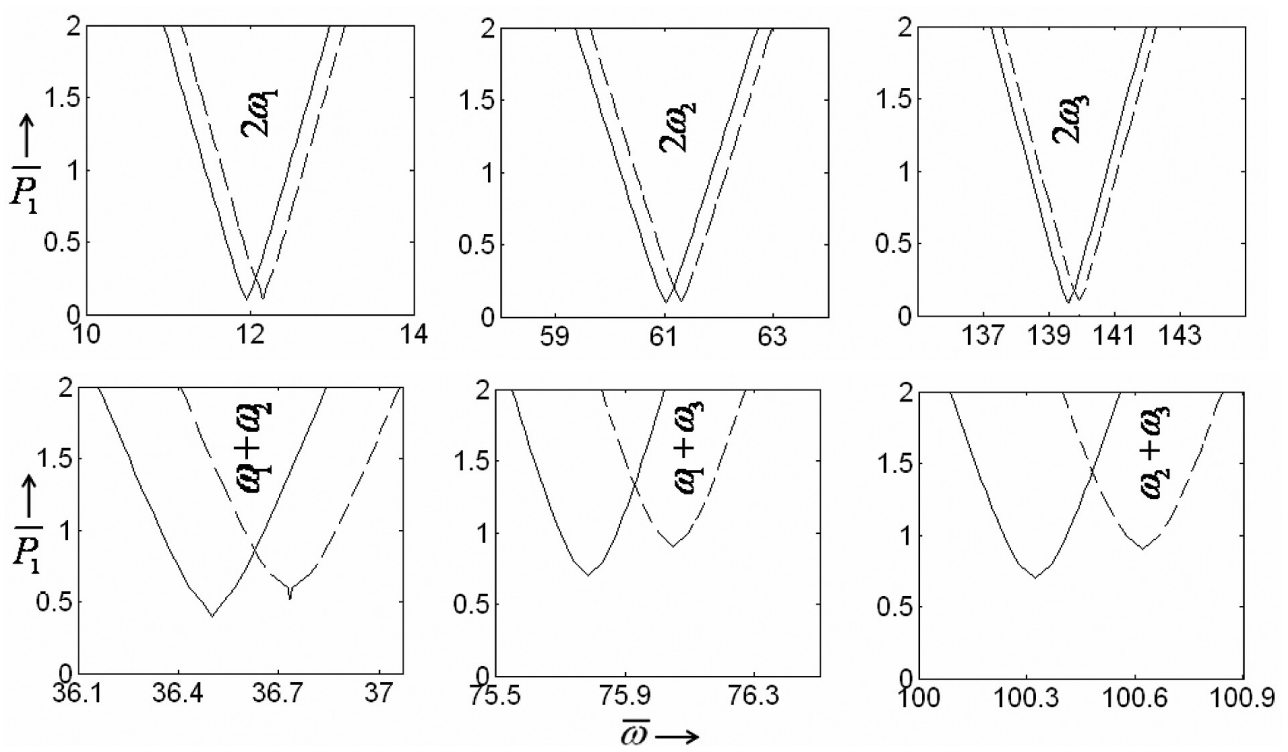

Fig. 16. Effect of shear parameter on instability regions for $\eta_{c}=0.1 .-g=0.0163,-\cdots-g=0.021$.

\subsection{Clamped-guided sandwich beam}

Figure 14 depicts the clamped guided (C-G) end conditions of the sandwich beam subjected to parametric excitation. The instability regions obtained in this case have been shown in Figs 15-19.

Figure 15 shows the instability regions for the sandwich beam with single MRE patch embedded in the middle of the foam type viscoelastic core sandwich beam. The instability regions for the first three mode principal parametric and combination parametric resonances of sum type have been shown here. In this case, the critical values of amplitude of excitation $\left(\bar{p}_{1 c r}\right)$ are 0.1 for first two modes and 0.09 for the third mode and the corresponding nondimensional 

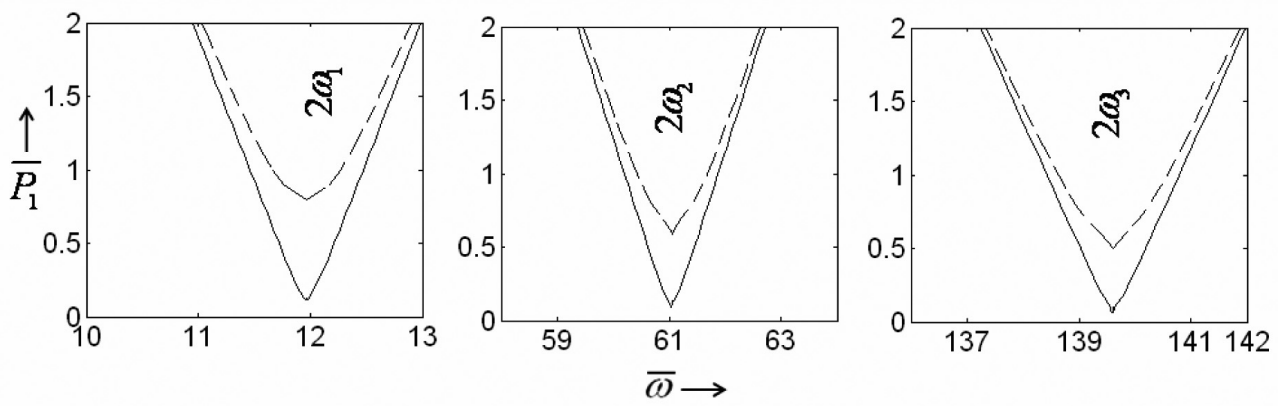

Fig. 17. Effect of core loss factor on instability regions for $g=0.0163$.
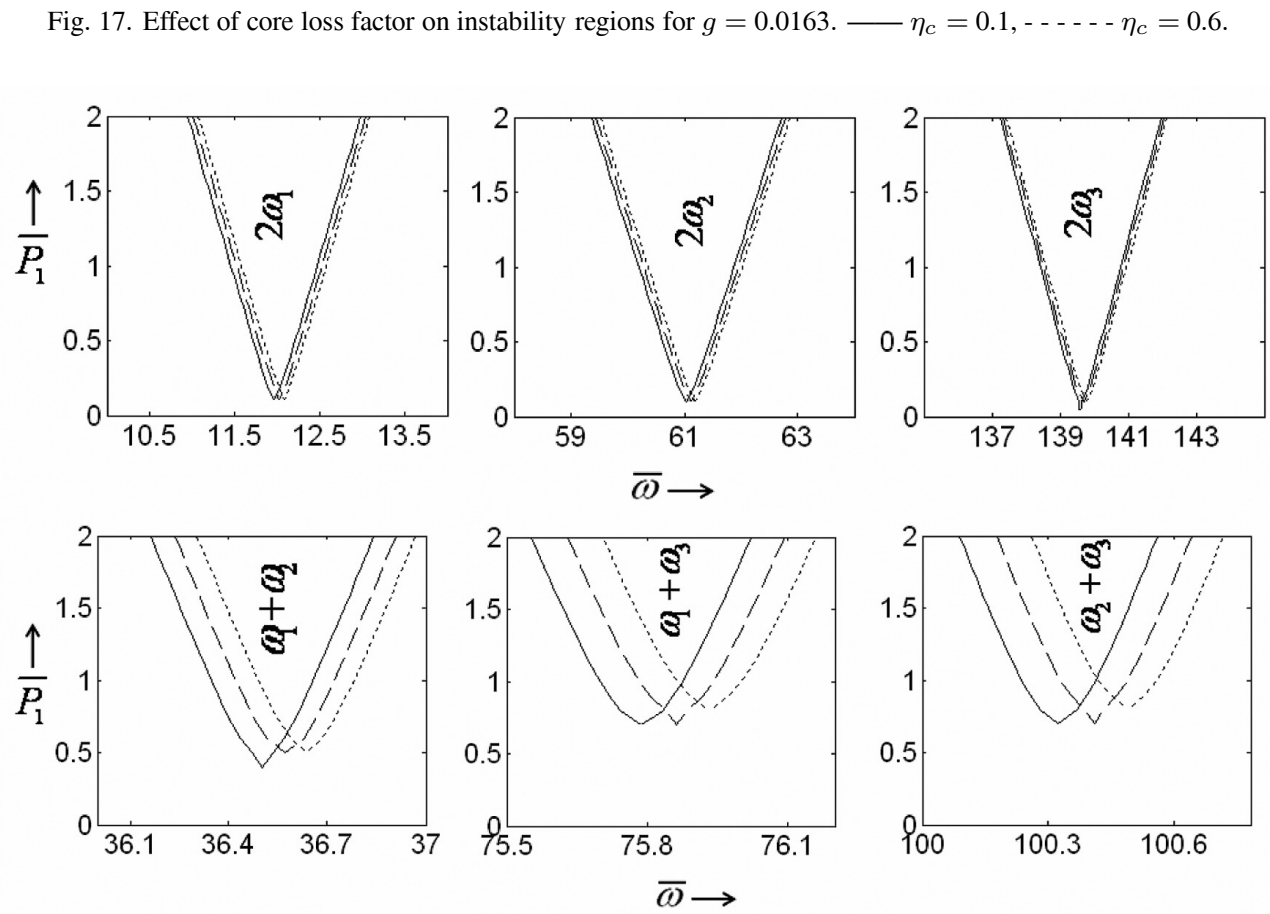

Fig. 18. Parametric instability regions for a sandwich beam with number of MRE patch equal to one (- - ), two (- - ) and three (- - - -) for principal parametric and combination parametric resonances of sum type.

frequency at which the system becomes unstable are 12 for the first mode, 61 for the second mode and 139.8 for the third mode. These values are less than those obtained for the simply supported and clamped pinned [24] boundary conditions.

Effect of shear parameter on the instability regions is shown in Fig. 16. Similar to the previous boundary condition, here also, with increase in shear parameter the instability regions shift towards right for both principal and combination parametric resonances. For principal parametric resonances with $g=0.021, \bar{P}_{1 \mathrm{cr}}$ values for the first three modes are 0.1 .

For combination resonances of sum type with $g=0.021$, the frequency values corresponding to $\bar{P}_{1 c r}$ are 36.5 for first and second modes, 75.6 for first and third mode and 100.4 for second and third modes. In combination resonances the $\bar{P}_{1 c r}$ value moves from 0.4 to 0.5 for first and second mode and 0.7 to 0.9 for second and third modes. Hence, unlike principal parametric resonance case, the stability of the system improves for combination resonance case with increase in shear parameter.

From Fig. 17 it is observed that instability region shifts upwards to higher amplitude with increase of core loss factor and the stability of the system is improved. With increase of core loss factor $\eta_{c}$ from 0.1 to 0.6 , the $\bar{P}_{1 \mathrm{cr}}$ value increases from 0.1 to 0.8 for first mode, 0.1 to 0.6 for second mode and 0.09 to 0.5 for third mode in case of 

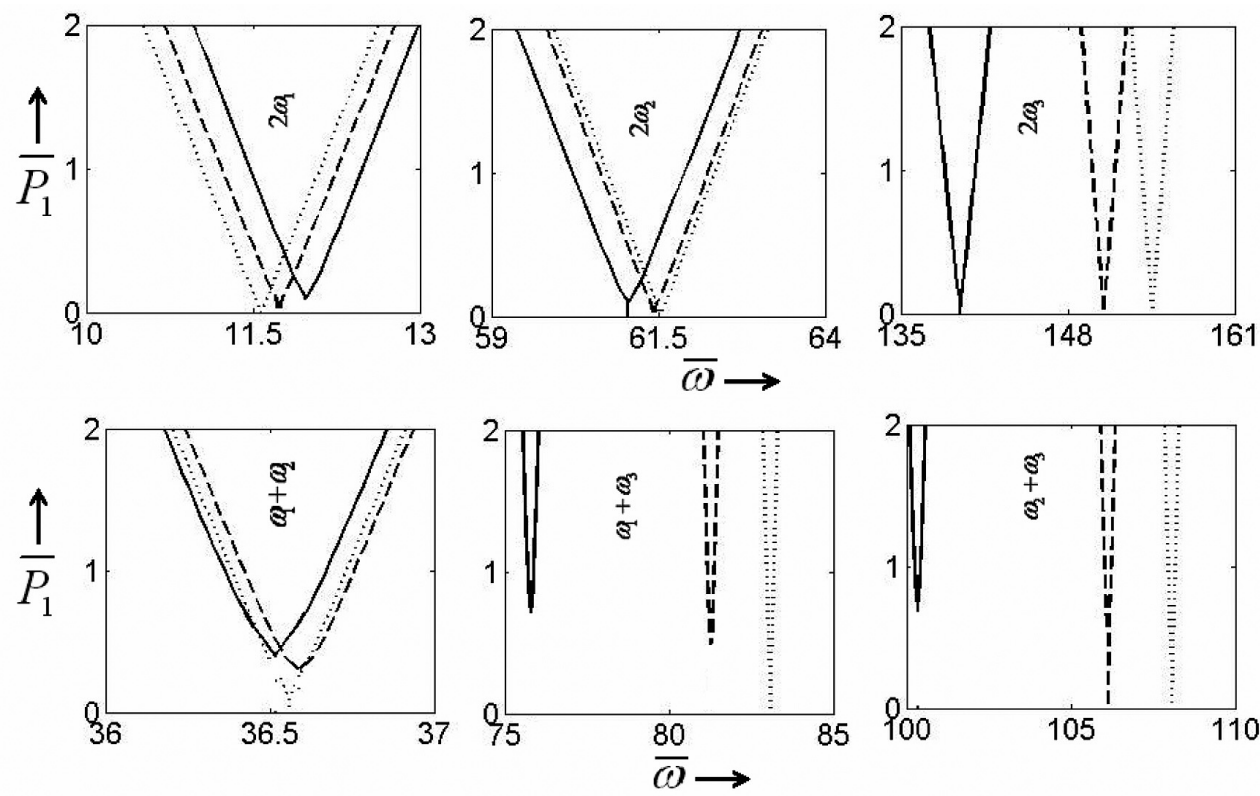

Fig. 19. Effect of variation of thickness of bottom layer on instability regions. $-d_{b}=10 \mathrm{~mm}, d_{b}=6 \mathrm{~mm},----d_{b}=2 \mathrm{~mm}$. Other parameters same as in Fig. 5 with $\bar{P}_{0}=0.1$.

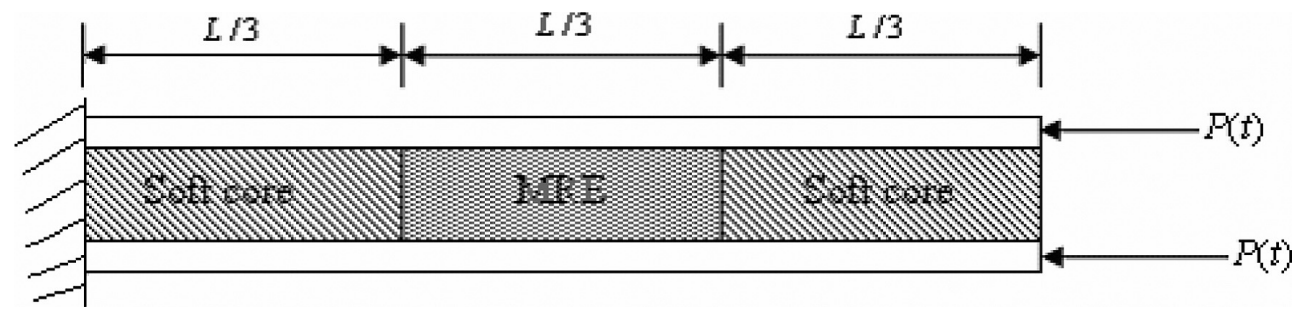

Fig. 20. Schematic diagram of MRE embedded soft cored sandwich beam with clamped -free end conditions subjected periodic axial load. (Dimensions not up to scale.).

principal parametric resonance. It may be noted that with increase in core loss factor to 0.6 the instability region for combination resonances disappears. Hence, the system becomes more stable by using core with higher loss factor.

The effect of increase in the number of MRE patches in the soft-core on the instability region has been shown in Fig. 18. Similar trends as in the previous boundary conditions have been observed. Here there is no appreciable change in $\bar{P}_{1 c r}$ value for principal parametric resonance and the region shifts to higher values of frequency with increase of MRE patches. With increase in number of MRE patches from one to three, for combination resonances of sum type $\bar{P}_{1 c r}$ value moves from 0.4 to 0.5 for combination of first and second modes, 0.7 to 0.8 for both combinations of second and third modes, and first and third modes.

Similar to the simply supported end conditions, in this case also the effects of the variation of bottom layer thickness on the instability region has been determined as shown in Fig. 19. In contrast to simply supported case, here instability regions for combination resonances have been observed in addition to the principal parametric resonances. Here also, with decrease in thickness while the instability region moves towards left for the first mode, for other two modes it moves towards right. This is due to the fact that the mode shapes in this case is similar to that of simply supported beam except at the ends. Due to the presence of both principal and combination parametric resonances, the system has a wide range of unstable zones in comparison to the simply supported case, and hence the operator should suitably choose the operating system parameters to operate in the stable region to avoid vibration of the system. 

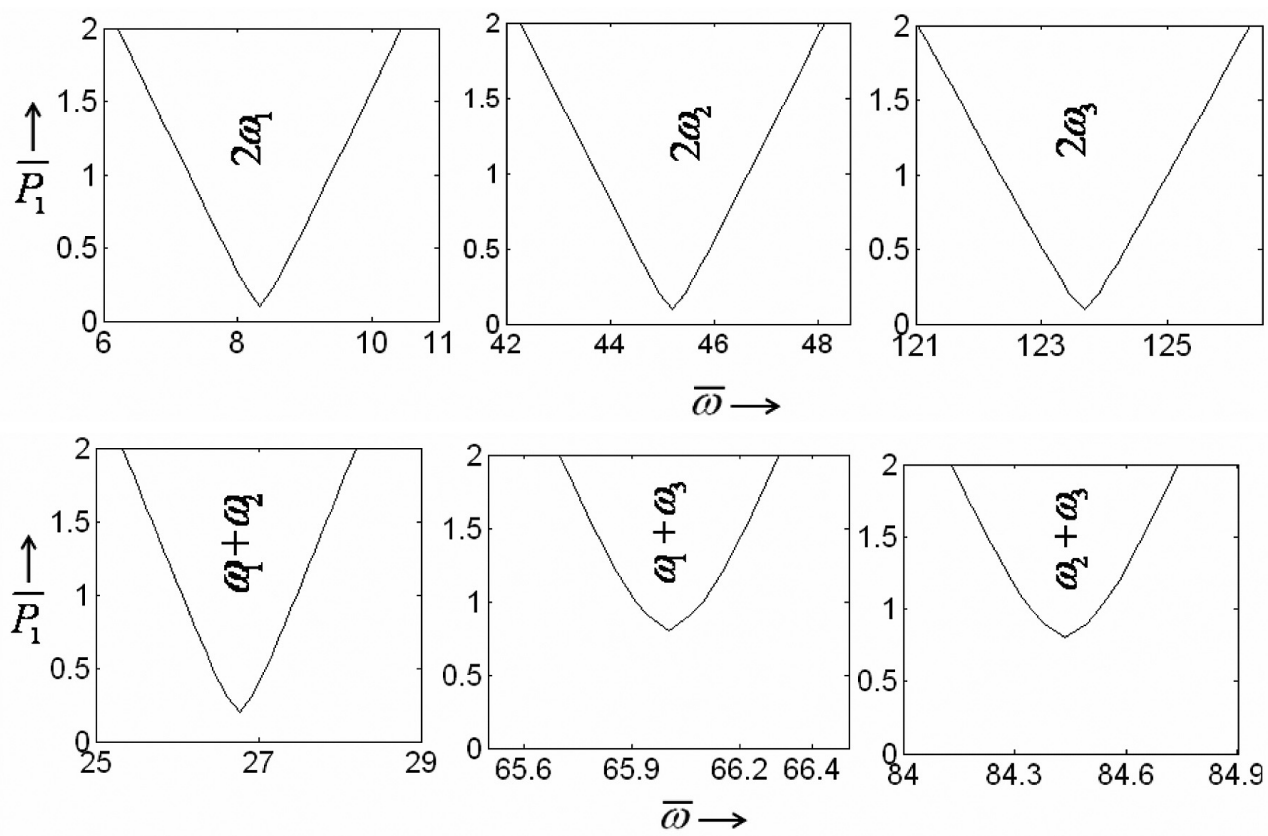

Fig. 21. Instability regions of C-F sandwich beam with alulight ${ }^{\circledR}$ skins and MRE placed in the middle of the soft-core; Key as in Fig. 5 with $\bar{P}_{0}=0.1$.
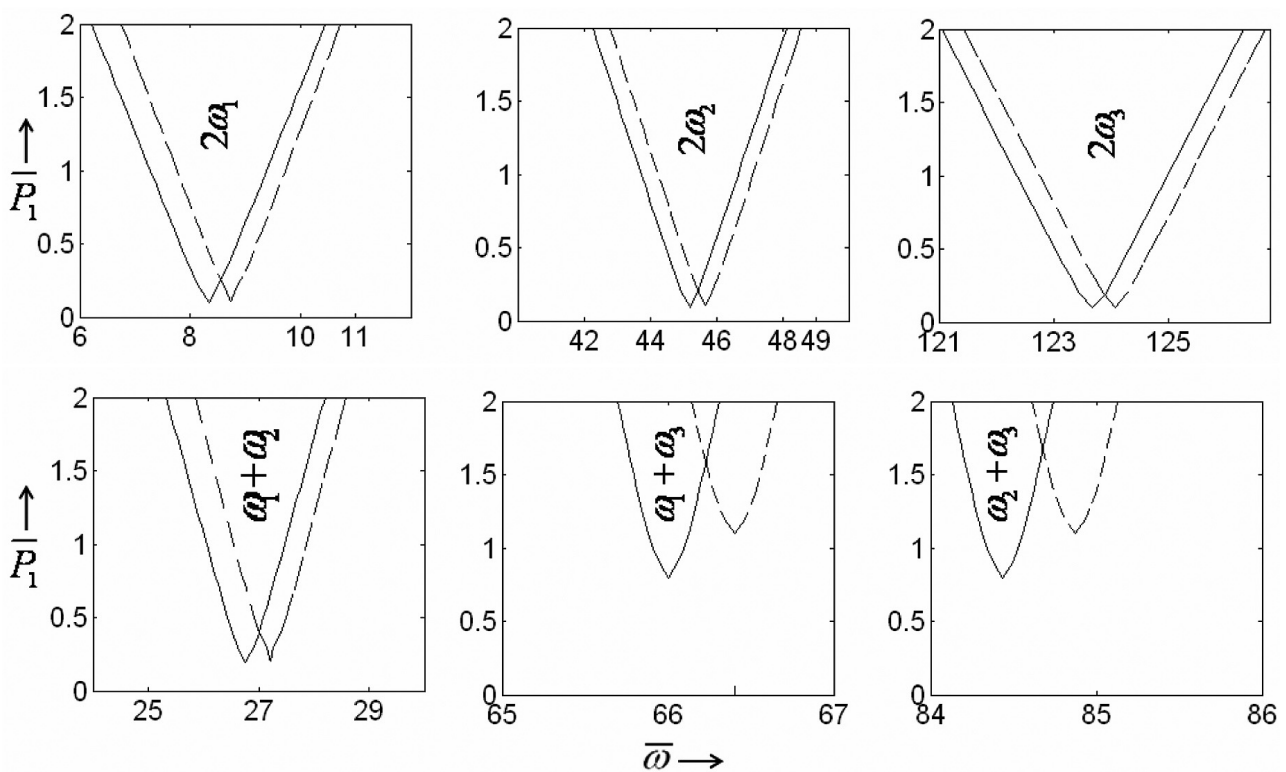

Fig. 22. Effect of shear parameter on instability regions for $\eta_{c}=0.1 .-g=0.0163,----g=0.021$.

\subsection{Clamped-free sandwich beam}

Figure 20 shows the schematic diagram of a sandwich beam subjected to parametric excitation with clamped free (C-F) end conditions. In this case the instability regions for the first three modes of vibration are determined and shown in Figs (21-25) for variation in different system parameters. 

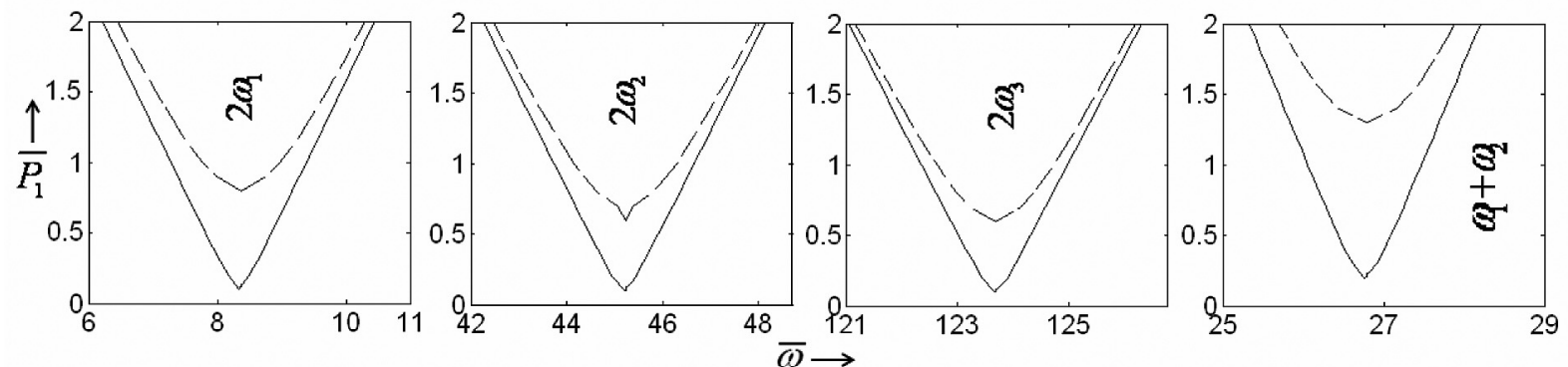

Fig. 23. Effect of core loss factor on instability regions for $g=0.0163 .-\eta_{c}=0.1,-\cdots \eta_{c}=0.6$.
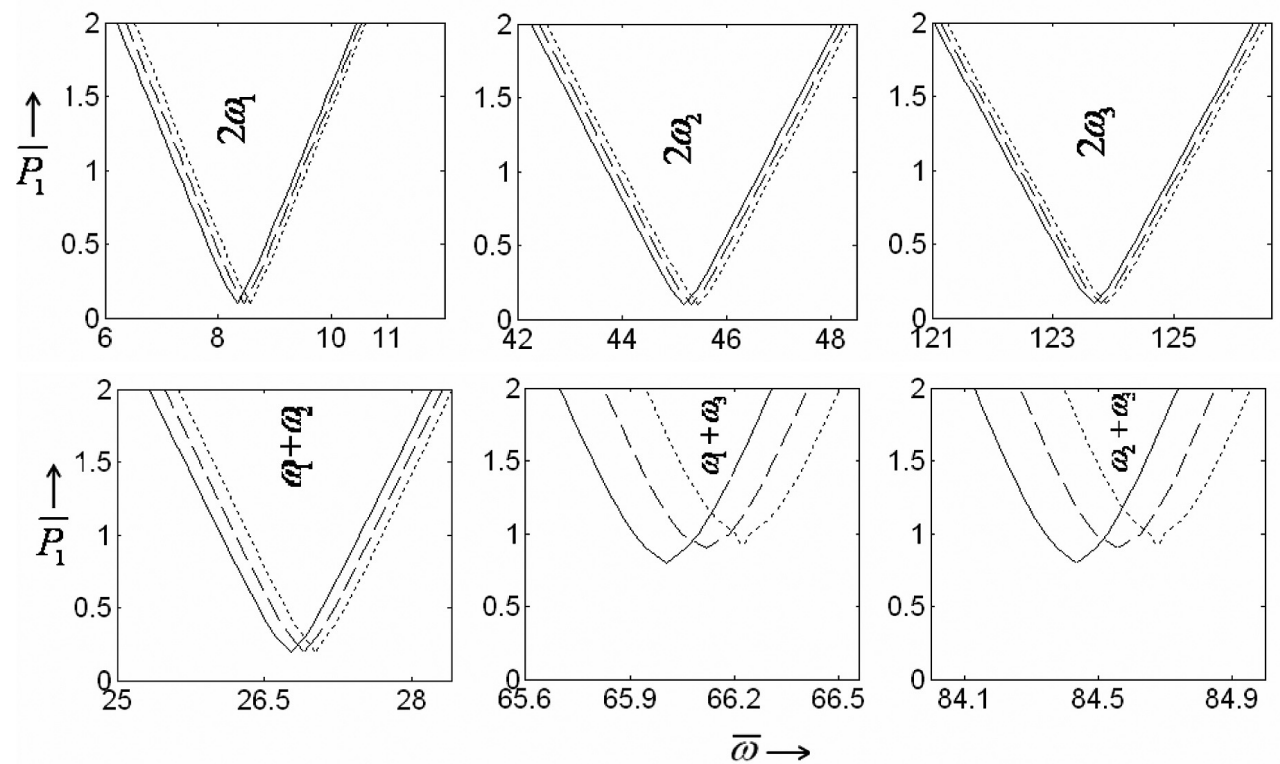

Fig. 24. Parametric instability regions for a sandwich beam with number of MRE patch equal to one (- - ), two (-- -) and three (- - - - - ) for principal and combination parametric resonances of sum type.

From the instability regions shown in Fig. 21, it is observed that the frequency values for which the system becomes unstable in this case are less than those obtained for other boundary conditions. The beam has been found to be stable for combination resonances of difference type. In this case the non dimensional frequency $\bar{\omega}$ for which the system has been found to be unstable for the critical value of $\bar{P}_{1 c r}$ is 8.2 for first mode, 45.4 for the second mode and 123.8 for the third mode.

The effect of increase in shear parameter has been shown in Fig. 22. With increase in shear parameter the instability region shifts towards right for first three modes. For principal parametric resonances, with increase in shear parameter $g$ to 0.021 , the non dimensional frequency at which the instability region starts for minimum values of $\bar{P}_{1 c r}$ is 8.5 for first mode, 45.9 for second mode and 124.3 for the third mode and the corresponding $\bar{P}_{1 c r}$ values are approximately same and equal to 0.1 for first three modes. For combination resonances of sum type increase in shear parameter improves the stability of the system significantly.

Figure 23 shows the effect of increase in core loss factor on instability regions. From these plots it may be observed that the instability region shifts upwards to higher amplitude of excitation with increase in core loss factor. The critical value $\bar{P}_{1 c r}$ increases from 0.1 to 0.7 for first mode, 0.1 to 0.6 for second and third modes with increase in $\eta_{c}$ from 0.1 to 0.6 . Unlike the previous cases, here, the instability regions have been observed for combination resonance of sum type for first and second modes with increase in the core loss factor. The system has been found 

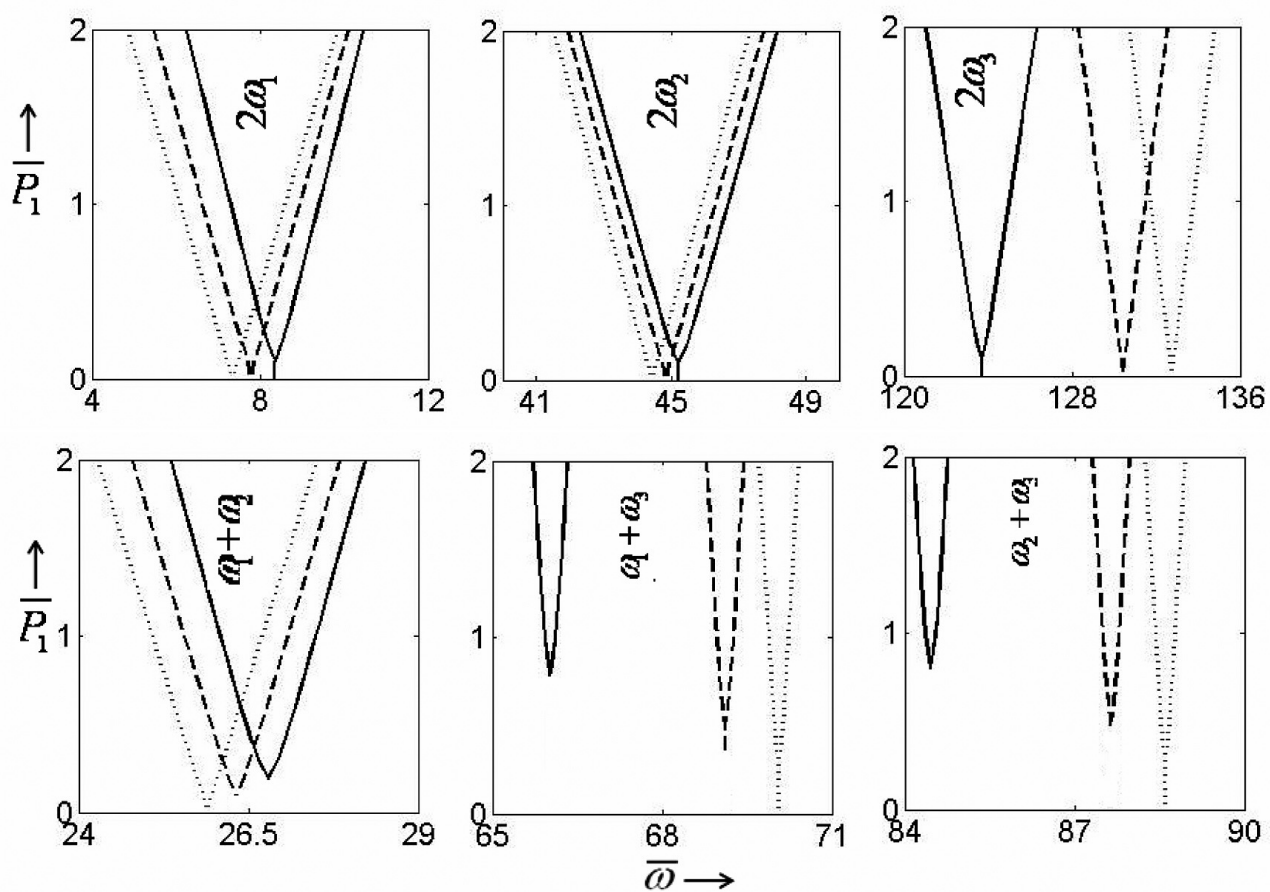

Fig. 25. Effect of variation of bottom layer thickness on instability regions. $-d_{b}=10 \mathrm{~mm},---d_{b}=6 \mathrm{~mm},----d_{b}=2 \mathrm{~mm}$. Other system parameters same as in Fig. 5 with $\bar{P}_{0}=0.1$.

to be stable for combination resonance of sum type for first and third, and second and third modes for higher values of core loss factor.

The effect of increase in the number of MRE patches in the soft-core on the instability regions have been studied using Fig. 24. The instability region shifts towards right with increase in the number of MRE patches. In combination resonance of sum type the instability region moves upwards also. It may be observed that while there is no appreciable change in the $\bar{P}_{1 c r}$ values for principal parametric resonances, in case of combination parametric resonances it increases with increase in the number of MRE patches.

The effect of change in the bottom layer thickness on the instability region is shown in Fig. 25 and clearly the trend in second mode in this case is different from the earlier two cases. In this case for the first two modes with decrease in the bottom layer thickness, the instability regions shift towards left and for the third mode it moves towards right. Though a node is formed in the second mode of the clamped-free boundary condition, due to the presence of the free end in this case, the increase in stiffness is not significant enough in comparison to other two boundaries studied above. This may be the reason for the occurrence of instability region at a lower frequency with decrease in the thickness for the second mode. Hence, instead of randomly guessing about the instability range to safely operate the system, one should properly calculate these regions using the given equations in this work.

\section{Conclusions}

In the present work, considering higher order theory, the governing equations of motion of single and multipatched-MRE embedded soft-cored unsymmetric sandwich beam with nonconductive skins subjected to periodic axial load have been derived using extended Hamilton's principle and generalized Galerkin's method. Here these equations are used to study the parametric instability regions, but one may use these equations for studying the free and forced vibration characteristics of MRE embedded soft cored symmetric and unsymmetric sandwich beams.

In this work, the parametric instability regions for principal parametric resonances, combination parametric resonances of sum and difference types up to third mode have been determined for simply-supported, clamped-guided 
and clamped-free boundary conditions. The effects of core loss factor, shear parameter, the number of MRE patches and variation of skin thickness on the instability regions have been studied. In all the cases, the critical amplitude below which the system will always remain at a stable trivial state has been determined. These values will be used as guidelines for the designer and operators of the sandwich structures.

For all boundary conditions, the sandwich beams are stable for combination resonance of difference type. With increase in shear parameter the instability region shifts towards right. Hence, the system will be unstable at a higher value of operating frequency. While, there is no appreciable change in the instability region for principal parametric resonances, for combination parametric resonances of sum types, the stability of the system improves with increase in shear parameter. With increase in core loss factor instability region decreases and also moves upwards. Hence, the system can operate at higher amplitude of excitation for a wide range of frequency without any vibration. For simply supported end conditions, beam is found to be stable for combination resonances. With increase in number of MRE patches as the shear parameter gets increased, the instability regions move to higher frequencies. While for all the three end-conditions considered here, increase in the thickness of one skin layer, shifts the first mode instability regions to a higher frequency and third mode instability region to a lower frequency, the second mode instability region of clamped-free boundary condition shows a different trend. Hence, it is difficult to intuitively predict the instability range and one should use the developed equations here to correctly predict the instability region to safely operate the system without any vibration.

Due to the use of foam type soft core to reduce the weight of the sandwich beam, the present analysis can be effectively used to attenuate the vibration actively or passively by changing the stiffness and core loss factor of the MRE embedded core materials. Depending on kinematic constraints and loading conditions, one may suitably choose the boundary condition to avoid unnecessary vibration and operate the system at a safe zone by operating the system at a load below the critical value of $\bar{P}_{1 c r}$. The developed expressions and instability plots will find application in the design of sandwich beam to reduce vibration of the structures with different boundary conditions.

\section{Appendix A}

The mass matrix is given by

$$
[M]=\left[\begin{array}{llll}
{\left[M_{11}\right]} & {\left[M_{12}\right]} & {\left[M_{13}\right]} & {\left[M_{14}\right]} \\
{\left[M_{21}\right]} & {\left[M_{22}\right]} & {\left[M_{23}\right]} & {\left[M_{24}\right]} \\
{\left[M_{31}\right]} & {\left[M_{32}\right]} & {\left[M_{33}\right]} & {\left[M_{34}\right]} \\
{\left[M_{41}\right]} & {\left[M_{42}\right]} & {\left[M_{43}\right]} & {\left[M_{44}\right]}
\end{array}\right]
$$

The stiffness matrix is given by $[K]=\left[K_{1}\right]-\bar{P}_{0}[F]$ where,

$$
\begin{aligned}
& {\left[K_{1}\right]=\left[\begin{array}{llll}
{\left[K_{11}\right]} & {\left[K_{12}\right]} & {\left[K_{13}\right]} & {\left[K_{14}\right]} \\
{\left[K_{21}\right]} & {\left[K_{22}\right]} & {\left[K_{23}\right]} & {\left[K_{24}\right]} \\
{\left[K_{31}\right]} & {\left[K_{32}\right]} & {\left[K_{33}\right]} & {\left[K_{34}\right]} \\
{\left[K_{41}\right]} & {\left[K_{42}\right]} & {\left[K_{43}\right]} & {\left[K_{44}\right]}
\end{array}\right]}
\end{aligned}
$$

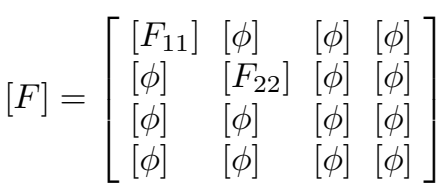

The elements of the various sub matrices are as follows

$$
\begin{aligned}
\left(M_{11}\right)_{i j}= & \left(\bar{m}_{t}\right)\left(\int_{0}^{1} w_{m i} w_{m j} d \bar{x}\right)+\bar{m}_{c} / 3\left(\int_{0}^{1} w_{m i} w_{m j} H_{1} d \bar{x}\right)+\left(\bar{m}_{r} / 3\right)\left(\int_{0}^{1} w_{m i} w_{m j} H_{2} d \bar{x}\right) \\
& +\left\{\left(\bar{m}_{c} / 12\right)\left(d_{t} / L\right)^{2}\right\}\left(\int_{0}^{1} w_{m i}^{\prime} w_{m j}^{\prime} H_{1} d \bar{x}\right)+\left\{\left(\bar{m}_{r} / 12\right)\left(d_{t} / L\right)^{2}\right\}\left(\int_{0}^{1} w_{m i}^{\prime} w_{m j}^{\prime} H_{2} d \bar{x}\right)
\end{aligned}
$$




$$
\begin{aligned}
& +\left\{\left(\bar{m}_{c} / 576\right)\left(d_{t} / c\right)\left(1+d_{t} / c\right)(c / L)^{4}\left(\xi_{c} / \phi_{c}\right)\right\}\left(\int_{0}^{1} w_{m i}^{\prime \prime} w_{m j}^{\prime \prime} H_{1} d \bar{x}\right) \\
& +\left\{\left(\bar{m}_{r} / 576\right)\left(d_{t} / c\right)\left(1+d_{t} / c\right)(c / L)^{4}\left(\xi_{r} / \phi_{r}\right)\right\}\left(\int_{0}^{1} w_{m i}^{\prime \prime} w_{m j}^{\prime \prime} H_{2} d \bar{x}\right) \\
& \left(M_{12}\right)_{i j}=\left(\bar{m}_{c} / 6\right)\left(\int_{0}^{1} w_{m i} w_{q j} H_{1} d \bar{x}\right)+\left(\bar{m}_{r} / 6\right)\left(\int_{0}^{1} w_{m i} w_{q j} H_{2} d \bar{x}\right) \\
& -\left\{\left(\bar{m}_{c} / 24\right)\left(d_{t} d_{b} / L^{2}\right)\right\}\left(\int_{0}^{1} w_{m i}^{\prime} w_{q j}^{\prime} H_{1} d \bar{x}\right)+\left\{\left(\bar{m}_{r} / 24\right)\left(d_{t} d_{b} / L^{2}\right)\right\}\left(\int_{0}^{1} w_{m j}^{\prime} w_{q i}^{\prime} H_{2} d \bar{x}\right) \\
& +\left\{\left(\bar{m}_{c} / 576\right)\left(d_{b} / c\right)\left(1+d_{t} / c\right)(c / L)^{4}\left(\xi_{c} / \phi_{c}\right)\right\}\left(\int_{0}^{1} w_{m i}^{\prime \prime} w_{q j}^{\prime \prime} H_{1} d \bar{x}\right) \\
& +\left\{\left(\bar{m}_{r} / 576\right)\left(d_{b} / c\right)\left(1+d_{t} / c\right)(c / L)^{4}\left(\xi_{r} / \phi_{r}\right)\right\}\left(\int_{0}^{1} w_{m i}^{\prime \prime} w_{q j}^{\prime \prime} H_{2} d \bar{x}\right) \\
& \left(M_{13}\right)_{i j}=-\left\{\left(\bar{m}_{t}\right)(1 / 48)\left(1+d_{t} / c\right)(c / L)^{3}\left(\xi_{c} / \phi_{c}\right)\right\}\left(\int_{0}^{1} w_{m i}^{\prime \prime} u_{r j}^{\prime} H_{1} d \bar{x}\right) \\
& -\left\{\left(\bar{m}_{t}\right)(1 / 48)\left(1+d_{t} / c\right)(c / L)^{3}\left(\xi_{r} / \phi_{r}\right)\right\}\left(\int_{0}^{1} w_{m i}^{\prime \prime} u_{r j}^{\prime} H_{2} d \bar{x}\right) \\
& +\left\{\left(\bar{m}_{c} / 288\right)\left(1+d_{t} / c\right)(c / L)^{3}\left(\xi_{c} / \phi_{c}\right)\right\}\left(\int_{0}^{1} w_{m i}^{\prime \prime} u_{r j}^{\prime} H_{1} d \bar{x}\right) \\
& +\left\{\left(\bar{m}_{r} / 288\right)\left(1+d_{t} / c\right)(c / L)^{3}\left(\xi_{r} / \phi_{r}\right)\right\}\left(\int_{0}^{1} w_{m i}^{\prime \prime} u_{r j}^{\prime} H_{2} d \bar{x}\right) \\
& -\left\{\left(\bar{m}_{c} / 6\right)\left(d_{t} / L\right)\right\}\left(\int_{0}^{1} w_{m i}^{\prime} u_{r j} H_{1} d \bar{x}\right)-\left\{\left(\bar{m}_{r} / 6\right)\left(d_{t} / L\right)\right\}\left(\int_{0}^{1} w_{m i}^{\prime} u_{r j} H_{2} d \bar{x}\right) \\
& \left(M_{14}\right)_{i j}=\left\{(1 / 48)\left(\bar{m}_{b}\right)\left(1+d_{t} / c\right)(c / L)^{3}\left(\xi_{c} / \phi_{c}\right)\right\}\left(\int_{0}^{1} w_{m i}^{\prime \prime} u_{s j}^{\prime} H_{1} d \bar{x}\right) \\
& +\left\{(1 / 48)\left(\bar{m}_{b}\right)\left(1+d_{t} / c\right)(c / L)^{3}\left(\xi_{r} / \phi_{r}\right)\right\}\left(\int_{0}^{1} w_{m i}^{\prime \prime} u_{s j}^{\prime} H_{2} d \bar{x}\right) \\
& +\left\{(1 / 48)\left(\bar{m}_{c} / 6\right)\left(1+d_{t} / c\right)(c / L)^{3}\left(\xi_{c} / \phi_{c}\right)\right\}\left(\int_{0}^{1} w_{m i}^{\prime \prime} u_{s j}^{\prime} H_{1} d \bar{x}\right)
\end{aligned}
$$




$$
\begin{aligned}
& +\left\{(1 / 48)\left(\bar{m}_{r} / 6\right)\left(1+d_{t} / c\right)(c / L)^{3}\left(\xi_{r} / \phi_{r}\right)\right\}\left(\int_{0}^{1} w_{m i}^{\prime \prime} u_{s j}^{\prime} H_{2} d \bar{x}\right) \\
& -\left\{\left(\bar{m}_{c} / 12\right)\left(d_{t} / L\right)\right\}\left(\int_{0}^{1} w_{m i}^{\prime} u_{s j} H_{1} d \bar{x}\right)-\left\{\left(\bar{m}_{r} / 12\right)\left(d_{t} / L\right)\right\}\left(\int_{0}^{1} w_{m i}^{\prime} u_{s j} H_{2} d \bar{x}\right) \\
& \left(M_{21}\right)_{i j}=\left(\bar{m}_{c} / 6\right)\left(\int_{0}^{1} w_{q i} w_{m j} H_{1} d \bar{x}\right)+\left(\bar{m}_{r} / 6\right)\left(\int_{0}^{1} w_{q i} w_{m j} H_{2} d \bar{x}\right) \\
& -\left\{\left(\bar{m}_{c} / 24\right)\left(d_{t} d_{b} / L^{2}\right)\right\}\left(\int_{0}^{1} w_{q i}^{\prime} w_{m j}^{\prime} H_{1} d \bar{x}\right)-\left\{\left(\bar{m}_{r} / 24\right)\left(d_{t} d_{b} / L^{2}\right)\right\}\left(\int_{0}^{1} w_{q i}^{\prime} w_{m j}^{\prime} H_{2} d \bar{x}\right) \\
& +\left\{\left(\bar{m}_{c} / 576\right)\left(d_{t} / c\right)\left(1+d_{b} / c\right)(c / L)^{4}\left(\xi_{c} / \phi_{c}\right)\right\}\left(\int_{0}^{1} w_{q i}^{\prime \prime} w_{m j}^{\prime \prime} H_{1} d \bar{x}\right) \\
& +\left\{\left(\bar{m}_{r} / 576\right)\left(d_{t} / c\right)\left(1+d_{b} / c\right)(c / L)^{4}\left(\xi_{r} / \phi_{r}\right)\right\}\left(\int_{0}^{1} w_{q i}^{\prime \prime} w_{m j}^{\prime \prime} H_{2} d \bar{x}\right) \\
& \left(M_{22}\right)_{i j}=\left(\bar{m}_{b}\right)\left(\int_{0}^{1} w_{q i} w_{q j} d \bar{x}\right)+\left(\bar{m}_{c} / 3\right)\left(\int_{0}^{1} w_{q i} w_{q j} H_{1} d \bar{x}\right)+\left(\bar{m}_{r} / 3\right)\left(\int_{0}^{1} w_{q i} w_{q j} H_{2} d \bar{x}\right) \\
& +\left\{\left(\bar{m}_{c} / 12\right)\left(d_{t} / L\right)^{2}\right\}\left(\int_{0}^{1} w_{q i}^{\prime} w_{q j}^{\prime} H_{1} d \bar{x}\right)+\left\{\left(\bar{m}_{r} / 12\right)\left(d_{t} / L\right)^{2}\right\}\left(\int_{0}^{1} w_{q i}^{\prime} w_{q j}^{\prime} H_{2} d \bar{x}\right) \\
& +\left\{\left(\bar{m}_{c} / 576\right)\left(d_{b} / c\right)\left(1+d_{b} / c\right)(c / L)^{4}\left(\xi_{c} / \phi_{c}\right)\right\}\left(\int_{0}^{1} w_{q i}^{\prime \prime} w_{q j}^{\prime \prime} H_{1} d \bar{x}\right) \\
& +\left\{\left(\bar{m}_{r} / 576\right)\left(d_{b} / c\right)\left(1+d_{b} / c\right)(c / L)^{4}\left(\xi_{r} / \phi_{r}\right)\right\}\left(\int_{0}^{1} w_{q i}^{\prime \prime} w_{q j}^{\prime \prime} H_{2} d \bar{x}\right) \\
& \left(M_{23}\right)_{i j}=-\left\{(1 / 48)\left(\bar{m}_{t}\right)\left(1+d_{b} / c\right)(c / L)^{3}\left(\xi_{c} / \phi_{c}\right)\right\}\left(\int_{0}^{1} w_{q i}^{\prime \prime} u_{r j}^{\prime} H_{1} d \bar{x}\right) \\
& -\left\{(1 / 48)\left(\bar{m}_{t}\right)\left(1+d_{b} / c\right)(c / L)^{3}\left(\xi_{r} / \phi_{r}\right)\right\}\left(\int_{0}^{1} w_{q i}^{\prime \prime} u_{r j}^{\prime} H_{2} d \bar{x}\right) \\
& -\left\{(1 / 48)\left(\bar{m}_{c} / 6\right)\left(1+d_{b} / c\right)(c / L)^{3}\left(\xi_{c} / \phi_{c}\right)\right\}\left(\int_{0}^{1} w_{q i}^{\prime \prime} u_{r j}^{\prime} H_{1} d \bar{x}\right) \\
& -\left\{(1 / 48)\left(\bar{m}_{r} / 6\right)\left(1+d_{b} / c\right)(c / L)^{3}\left(\xi_{r} / \phi_{r}\right)\right\}\left(\int_{0}^{1} w_{q i}^{\prime \prime} u_{r j}^{\prime} H_{2} d \bar{x}\right)
\end{aligned}
$$




$$
\begin{aligned}
& +\left\{\left(m_{c} / 12\right)\left(d_{b} / L\right)\right\}\left(\int_{0}^{1} w_{q i}^{\prime} u_{r j} H_{1} d \bar{x}\right)+\left\{\left(m_{r} / 12\right)\left(d_{b} / L\right)\right\}\left(\int_{0}^{1} w_{q i}^{\prime} u_{r j} H_{2} d \bar{x}\right) \\
& \left(M_{24}\right)_{i j}=\left\{(1 / 48)\left(\bar{m}_{b}\right)\left(1+d_{b} / c\right)(c / L)^{3}\left(\xi_{c} / \phi_{c}\right)\right\}\left(\int_{0}^{1} w_{q i}^{\prime \prime} u_{s j}^{\prime} H_{1} d \bar{x}\right) \\
& +\left\{(1 / 48)\left(\bar{m}_{b}\right)\left(1+d_{b} / c\right)(c / L)^{3}\left(\xi_{r} / \phi_{r}\right)\right\}\left(\int_{0}^{1} w_{q i}^{\prime \prime} u_{s j}^{\prime} H_{2} d \bar{x}\right) \\
& +\left\{(1 / 48)\left(\bar{m}_{c} / 6\right)\left(1+d_{b} / c\right)(c / L)^{3}\left(\xi_{c} / \phi_{c}\right)\right\}\left(\int_{0}^{1} w_{q i}^{\prime \prime} u_{s j}^{\prime} H_{1} d \bar{x}\right) \\
& +\left\{(1 / 48)\left(\bar{m}_{r} / 6\right)\left(1+d_{b} / c\right)(c / L)^{3}\left(\xi_{r} / \phi_{r}\right)\right\}\left(\int_{0}^{1} w_{q i}^{\prime \prime} u_{s j}^{\prime} H_{2} d \bar{x}\right) \\
& +\left(m_{c} / 6\right)\left(d_{b} / L\right)\left(\int_{0}^{1} w_{q i}^{\prime} u_{s j} H_{1} d \bar{x}\right)+\left(m_{r} / 6\right)\left(d_{b} / L\right)\left(\int_{0}^{1} w_{q i}^{\prime} u_{s j} H_{2} d \bar{x}\right) \\
& \left(M_{31}\right)_{i j}=-\left\{\left(m_{c} / 288\right)\left(d_{t} / c\right)(c / L)^{3}\left(\xi_{c} / \phi_{c}\right)\right\}\left(\int_{0}^{1} u_{r i}^{\prime \prime} w_{m j}^{\prime} H_{1} d \bar{x}\right) \\
& -\left\{\left(m_{r} / 288\right)\left(d_{t} / c\right)(c / L)^{3}\left(\xi_{r} / \phi_{r}\right)\right\}\left(\int_{0}^{1} u_{r i}^{\prime \prime} w_{m j}^{\prime} H_{2} d \bar{x}\right) \\
& -\left\{\left(\bar{m}_{c} / 6\right)\left(d_{t} / L\right)\right\}\left(\int_{0}^{1} u_{r i}^{\prime} w_{m j} H_{1} d \bar{x}\right)-\left\{\left(\bar{m}_{r} / 6\right)\left(d_{t} / L\right)\right\}\left(\int_{0}^{1} u_{r i}^{\prime} w_{m j} H_{2} d \bar{x}\right) \\
& \left(M_{32}\right)_{i j}=\left\{\left(\bar{m}_{c} / 12\right)\left(d_{b} / L\right)\right\}\left(\int_{0}^{1} u_{r i}^{\prime} w_{q j} H_{1} d \bar{x}\right)+\left\{\left(\bar{m}_{r} / 12\right)\left(d_{b} / L\right)\right\}\left(\int_{0}^{1} u_{r i}^{\prime} w_{q j} H_{2} d \bar{x}\right) \\
& -\left\{\left(\bar{m}_{c} / 288\right)\left(d_{b} / c\right)(c / L)^{3}\left(\xi_{c} / \phi_{c}\right)\right\}\left(\int_{0}^{1} u_{r i}^{\prime \prime} w_{q j}^{\prime} H_{1} d \bar{x}\right) \\
& -\left\{\left(\bar{m}_{r} / 288\right)\left(d_{b} / c\right)(c / L)^{3}\left(\xi_{r} / \phi_{r}\right)\right\}\left(\int_{0}^{1} u_{r i}^{\prime \prime} w_{q j}^{\prime} H_{2} d \bar{x}\right) \\
& \left(M_{33}\right)_{i j}=-\left\{(1 / 24)\left(\bar{m}_{t}\right)(c / L)^{2}\left(\xi_{c} / \phi_{c}\right)\right\}\left(\int_{0}^{1} u_{r i}^{\prime} u_{r j}^{\prime} H_{1} d \bar{x}\right) \\
& -\left\{(1 / 24)\left(\bar{m}_{t}\right)(c / L)^{2}\left(\xi_{r} / \phi_{r}\right)\right\}\left(\int_{0}^{1} u_{r i}^{\prime} u_{r j}^{\prime} H_{2} d \bar{x}\right)
\end{aligned}
$$


782

S.K. Dwivedy and M. Srinivas / Dynamic instability of MRE embedded soft cored sandwich beam with non-conductive skins

$$
\begin{aligned}
& -\left\{(1 / 24)\left(\bar{m}_{c} / 6\right)(c / L)^{2}\left(\xi_{c} / \phi_{c}\right)\right\}\left(\int_{0}^{1} u_{r i}^{\prime} u_{r j}^{\prime} H_{1} d \bar{x}\right)-\left\{(1 / 24)\left(\bar{m}_{r} / 6\right)(c / L)^{2}\left(\xi_{r} / \phi_{r}\right)\right\} \\
& \left(\int_{0}^{1} u_{r i}^{\prime} u_{r j}^{\prime} H_{2} d \bar{x}\right) \\
& -\left(\bar{m}_{t}\right)\left(\int_{0}^{L} u_{r i} u_{r j} d \bar{x}\right)-\left(\bar{m}_{c} / 3\right)\left(\int_{0}^{1} u_{r i} u_{r j} H_{1} d \bar{x}\right)-\left(\bar{m}_{c} / 3\right)\left(\int_{0}^{1} u_{r i} u_{r j} H_{2} d \bar{x}\right) \\
& \left(M_{34}\right)_{i j}=-\left(\bar{m}_{c} / 6\right)\left(\int_{0}^{1} u_{r i} u_{s j} H_{1} d \bar{x}\right)-\left(\bar{m}_{r} / 6\right)\left(\int_{0}^{1} u_{r i} u_{s j} H_{2} d \bar{x}\right) \\
& +\left\{\left(\bar{m}_{b} / 24\right)(c / L)^{2}\left(\xi_{c} / \phi_{c}\right)\right\}\left(\int_{0}^{1} u_{r i}^{\prime} u_{s j}^{\prime} H_{1} d \bar{x}\right)+\left\{\left(\bar{m}_{b} / 24\right)(c / L)^{2}\left(\xi_{r} / \phi_{r}\right)\right\} \\
& \left(\int_{0}^{1} u_{r i}^{\prime} u_{s j}^{\prime} H_{2} d \bar{x}\right) \\
& +\left\{\left(\bar{m}_{c} / 144\right)(c / L)^{2}\left(\xi_{c} / \phi_{c}\right)\right\}\left(\int_{0}^{1} u_{r i}^{\prime} u_{s j}^{\prime} H_{1} d \bar{x}\right)+\left\{\left(\bar{m}_{c} / 144\right)(c / L)^{2}\left(\xi_{r} / \phi_{r}\right)\right\} \\
& \left(\int_{0}^{1} u_{r i}^{\prime} u_{s j}^{\prime} H_{2} d \bar{x}\right) \\
& \left(M_{41}\right)_{i j}=-\left\{\left(\bar{m}_{c} / 12\right)\left(d_{t} / L\right)\right\}\left(\int_{0}^{1} u_{s i}^{\prime} w_{m j} H_{1} d \bar{x}\right)-\left\{\left(\bar{m}_{r} / 12\right)\left(d_{t} / L\right)\right\}\left(\int_{0}^{1} u_{s i}^{\prime} w_{m j} H_{2} d \bar{x}\right) \\
& +\left\{\left(\bar{m}_{c} / 288\right)\left(d_{t} / c\right)(c / L)^{3}\left(\xi_{c} / \phi_{c}\right)\right\}\left(\int_{0}^{1} u_{s i}^{\prime \prime} w_{m j}^{\prime} H_{1} d \bar{x}\right) \\
& +\left\{\left(\bar{m}_{r} / 288\right)\left(d_{t} / c\right)(c / L)^{3}\left(\xi_{r} / \phi_{r}\right)\right\}\left(\int_{0}^{1} u_{s i}^{\prime \prime} w_{m j}^{\prime} H_{2} d \bar{x}\right) \\
& \left(M_{42}\right)_{i j}=\left\{\left(\bar{m}_{c} / 6\right)\left(d_{b} / L\right)\right\}\left(\int_{0}^{1} u_{s i}^{\prime} w_{q j} H_{1} d \bar{x}\right)+\left\{\left(\bar{m}_{r} / 6\right)\left(d_{b} / L\right)\right\}\left(\int_{0}^{1} u_{s i}^{\prime} w_{q j} H_{2} d \bar{x}\right) \\
& +\left\{\left(\bar{m}_{c} / 288\right)\left(d_{b} / c\right)(c / L)^{3}\left(\xi_{c} / \phi_{c}\right)\right\}\left(\int_{0}^{1} u_{s i}^{\prime \prime} w_{q j}^{\prime} H_{1} d \bar{x}\right) \\
& +\left\{\left(\bar{m}_{r} / 288\right)\left(d_{b} / c\right)(c / L)^{3}\left(\xi_{r} / \phi_{r}\right)\right\}\left(\int_{0}^{1} u_{s i}^{\prime \prime} w_{q j}^{\prime} H_{2} d \bar{x}\right)
\end{aligned}
$$




$$
\begin{aligned}
& \left(M_{43}\right)_{i j}=-\left(\bar{m}_{c} / 6\right)\left(\int_{0}^{1} u_{s i} u_{r j} H_{1} d \bar{x}\right)-\left(\bar{m}_{r} / 6\right)\left(\int_{0}^{1} u_{s i} u_{r j} H_{2} d \bar{x}\right) \\
& +\left\{\left(\bar{m}_{t} / 24\right)(c / L)^{2}\left(\xi_{c} / \phi_{c}\right)\right\}\left(\int_{0}^{1} u_{s i}^{\prime} u_{r j}^{\prime} H_{1} d \bar{x}\right)+\left\{\left(\bar{m}_{t} / 24\right)(c / L)^{2}\left(\xi_{r} / \phi_{r}\right)\right\} \\
& \left(\int_{0}^{1} u_{s i}^{\prime} u_{r j}^{\prime} H_{2} d \bar{x}\right) \\
& +\left\{\left(\bar{m}_{c} / 144\right)(c / L)^{2}\left(\xi_{c} / \phi_{c}\right)\right\}\left(\int_{0}^{1} u_{s i}^{\prime} u_{r j}^{\prime} H_{1} d \bar{x}\right)+\left\{\left(\bar{m}_{r} / 144\right)(c / L)^{2}\left(\xi_{r} / \phi_{r}\right)\right\} \\
& \left(\int_{0}^{1} u_{s i}^{\prime} u_{r j}^{\prime} H_{2} d \bar{x}\right) \\
& \left(M_{44}\right)_{i j}=-\left(\bar{m}_{b}\right)\left(\int_{0}^{1} u_{s i} u_{s j} d \bar{x}\right)-\left(\bar{m}_{c} / 3\right)\left(\int_{0}^{1} u_{s i} u_{s j} H_{1} d \bar{x}\right)-\left(\bar{m}_{r} / 3\right)\left(\int_{0}^{1} u_{s i} u_{s j} H_{2} d \bar{x}\right) \\
& -\left\{\left(\bar{m}_{b} / 24\right)(c / L)^{2}\left(\xi_{c} / \phi_{c}\right)\right\}\left(\int_{0}^{1} u_{s i}^{\prime} u_{s j}^{\prime} H_{1} d \bar{x}\right)-\left\{\left(\bar{m}_{b} / 24\right)(c / L)^{2}\left(\xi_{r} / \phi_{r}\right)\right\} \\
& \left(\int_{0}^{1} u_{s i}^{\prime} u_{s j}^{\prime} H_{2} d \bar{x}\right) \\
& -\left\{\left(\bar{m}_{c} / 144\right)(c / L)^{2}\left(\xi_{c} / \phi_{c}\right)\right\}\left(\int_{0}^{1} u_{s i}^{\prime} u_{s j}^{\prime} H_{1} d \bar{x}\right)-\left\{\left(\bar{m}_{r} / 144\right)(c / L)^{2}\left(\xi_{r} / \phi_{r}\right)\right\} \\
& \left(\int_{0}^{1} u_{s i}^{\prime} u_{s j}^{\prime} H_{2} d \bar{x}\right) \\
& \left(K_{11}\right)_{i j}=\left\{\left(\xi_{c} / 4\right)\left(1+d_{t} / c\right)^{2}\right\}\left(\int_{0}^{1} w_{m i}^{\prime} w_{m j}^{\prime} H_{1} d \bar{x}\right)+\left\{\left(\xi_{r} / 4\right)\left(1+d_{t} / c\right)^{2}\right\}\left(\int_{0}^{1} w_{m i}^{\prime} w_{m j}^{\prime} H_{2} d \bar{x}\right) \\
& +\left\{\left(\phi_{t} / 12\right)\left(d_{t} / L\right)^{2}\right\}\left(\int_{0}^{1} w_{m i}^{\prime \prime} w_{m j}^{\prime \prime} d \bar{x}\right)+\left\{\phi_{c}(L / c)^{2}\right\}\left(\int_{0}^{1} w_{m i} w_{m j} d \bar{x}\right) \\
& \left(K_{12}\right)_{i j}=-\left\{\phi_{c}(L / c)^{2}\right\}\left(\int_{0}^{1} w_{m i} w_{q j} d \bar{x}\right) \\
& +\left\{\left(\xi_{c} / 4\right)\left(1+d_{t} / c\right)\left(1+d_{b} / c\right)\right\}\left(\int_{0}^{1} w_{m i}^{\prime} w_{q j}^{\prime} H_{1} d \bar{x}\right)+\left\{\left(\xi_{r} / 4\right)\left(1+d_{t} / c\right)\left(1+d_{b} / c\right)\right\}
\end{aligned}
$$




$$
\begin{aligned}
& \left(\int_{0}^{1} w_{m i}^{\prime} w_{q j}^{\prime} H_{2} d \bar{x}\right) \\
& \left(K_{13}\right)_{i j}=-\left\{\left(\xi_{c} / 2\right)(L / c)\left(1+d_{t} / c\right)\right\}\left(\int_{0}^{1} w_{m i}^{\prime} u_{r j} H_{1} d \bar{x}\right)-\left\{\left(\xi_{r} / 2\right)(L / c)\left(1+d_{t} / c\right)\right\} \\
& \left(\int_{0}^{1} w_{m i}^{\prime} u_{r j} H_{2} d \bar{x}\right) \\
& -\left\{\left(\phi_{t} / 48\right)(c / L)^{3}\left(1+d_{t} / c\right)\left(\xi_{c} / \phi_{c}\right)\right\}\left(\int_{0}^{1} w_{m i}^{\prime \prime \prime} u_{r j}^{\prime \prime} H_{1} d \bar{x}\right) \\
& -\left\{\left(\phi_{t} / 48\right)(c / L)^{3}\left(1+d_{t} / c\right)\left(\xi_{r} / \phi_{r}\right)\right\}\left(\int_{0}^{1} w_{m i}^{\prime \prime \prime} u_{r j}^{\prime \prime} H_{2} d \bar{x}\right) \\
& \left(K_{14}\right)_{i j}=\left\{\left(\xi_{c} / 2\right)(L / c)\left(1+d_{t} / c\right)\right\}\left(\int_{0}^{1} w_{m i}^{\prime} u_{s j} H_{1} d \bar{x}\right)+\left\{\left(\xi_{r} / 2\right)(L / c)\left(1+d_{t} / c\right)\right\}\left(\int_{0}^{1} w_{m i}^{\prime} u_{s j} H_{2} d \bar{x}\right) \\
& +\left\{\left(\phi_{b} / 48\right)(c / L)^{3}\left(1+d_{t} / c\right)\left(\xi_{c} / \phi_{c}\right)\right\}\left(\int_{0}^{1} w_{m i}^{\prime \prime \prime} u_{s j}^{\prime \prime} H_{1} d \bar{x}\right) \\
& +\left\{\left(\phi_{b} / 48\right)(c / L)^{3}\left(1+d_{t} / c\right)\left(\xi_{r} / \phi_{r}\right)\right\}\left(\int_{0}^{1} w_{m i}^{\prime \prime \prime} u_{s j}^{\prime \prime} H_{2} d \bar{x}\right) \\
& \left(K_{21}\right)_{i j}=-\left\{\phi_{c}(L / c)^{2}\right\}\left(\int_{0}^{1} w_{q i} w_{m j} d \bar{x}\right)+\left\{\left(\xi_{c} / 4\right)\left(1+d_{t} / c\right)\left(1+d_{b} / c\right)\right\}\left(\int_{0}^{1} w_{q i}^{\prime} w_{m j}^{\prime} H_{1} d \bar{x}\right) \\
& +\left\{\left(\xi_{r} / 4\right)\left(1+d_{t} / c\right)\left(1+d_{b} / c\right)\right\}\left(\int_{0}^{1} w_{q i}^{\prime} w_{m j}^{\prime} H_{2} d \bar{x}\right) \\
& \left(K_{22}\right)_{i j}=\left\{\left(\xi_{c} / 4\right)\left(1+d_{b} / c\right)^{2}\right\}\left(\int_{0}^{1} w_{q i}^{\prime} w_{q j}^{\prime} H_{1} d \bar{x}\right)+\left\{\left(\xi_{r} / 4\right)\left(1+d_{b} / c\right)^{2}\right\}\left(\int_{0}^{1} w_{q i}^{\prime} w_{q j}^{\prime} H_{2} d \bar{x}\right) \\
& +\left\{\left(\phi_{b} / 12\right)\left(d_{b} / L\right)^{2}\right\}\left(\int_{0}^{1} w_{q i}^{\prime \prime} w_{q j}^{\prime \prime} d \bar{x}\right) \\
& \left(K_{23}\right)_{i j}=\left\{\left(-\xi_{c} / 2\right)(L / c)\left(1+d_{b} / c\right)\right\}\left(\int_{0}^{1} w_{q i}^{\prime} u_{r j} H_{1} d \bar{x}\right)+\left\{\left(-\xi_{r} / 2\right)(L / c)\left(1+d_{b} / c\right)\right\}\left(\int_{0}^{1} w_{q i}^{\prime} u_{r j} H_{2} d \bar{x}\right) \\
& -\left\{\left(\phi_{t} / 48\right)(c / L)^{3}\left(1+d_{b} / c\right)\left(\xi_{c} / \phi_{c}\right)\right\}\left(\int_{0}^{1} w_{q i}^{\prime \prime \prime} u_{r j}^{\prime \prime} H_{1} d \bar{x}\right)
\end{aligned}
$$


S.K. Dwivedy and M. Srinivas / Dynamic instability of MRE embedded soft cored sandwich beam with non-conductive skins

785

$$
\begin{aligned}
& -\left\{\left(\phi_{t} / 48\right)(c / L)^{3}\left(1+d_{b} / c\right)\left(\xi_{r} / \phi_{r}\right)\right\}\left(\int_{0}^{1} w_{q i}^{\prime \prime \prime} u_{r j}^{\prime \prime} H_{2} d \bar{x}\right) \\
& \left(K_{24}\right)_{i j}=\left\{\left(\xi_{c} / 2\right)(L / c)\left(1+d_{b} / c\right)\right\}\left(\int_{0}^{1} w_{q i}^{\prime} u_{s j} H_{1} d \bar{x}\right)+\left\{\left(\xi_{r} / 2\right)(L / c)\left(1+d_{b} / c\right)\right\}\left(\int_{0}^{1} w_{q i}^{\prime} u_{s j} H_{2} d \bar{x}\right) \\
& +\left\{\left(\phi_{b} / 48\right)(c / L)^{3}\left(1+d_{b} / c\right)\left(\xi_{c} / \phi_{c}\right)\right\}\left(\int_{0}^{1} w_{q i}^{\prime \prime \prime} u_{s j}^{\prime \prime} H_{1} d \bar{x}\right) \\
& +\left\{\left(\phi_{b} / 48\right)(c / L)^{3}\left(1+d_{b} / c\right)\left(\xi_{r} / \phi_{r}\right)\right\}\left(\int_{0}^{1} w_{q i}^{\prime \prime \prime} u_{s j}^{\prime \prime} H_{2} d \bar{x}\right) \\
& \left(K_{31}\right)_{i j}=\left\{\left(-\xi_{c} / 2\right)(L / c)\left(1+d_{t} / c\right)\right\}\left(\int_{0}^{1} u_{r i}^{\prime} w_{m j} H_{1} d \bar{x}\right)+\left\{\left(-\xi_{r} / 2\right)(L / c)\left(1+d_{t} / c\right)\right\} \\
& \left(\int_{0}^{1} u_{r i}^{\prime} w_{m j} H_{2} d \bar{x}\right) \\
& \left(K_{32}\right)_{i j}=\left\{\left(-\xi_{c} / 2\right)(L / c)\left(1+d_{b} / c\right)\right\}\left(\int_{0}^{1} u_{r i}^{\prime} w_{q j} H_{1} d \bar{x}\right)+\left\{\left(-\xi_{r} / 2\right)(L / c)\left(1+d_{b} / c\right)\right\} \\
& \left(\int_{0}^{1} u_{r i}^{\prime} w_{q j} H_{2} d \bar{x}\right) \\
& \left(K_{33}\right)_{i j}=\left(-\phi_{t}\right)\left(\int_{0}^{1} u_{r i}^{\prime} u_{r j}^{\prime} d \bar{x}\right)-\left\{(L / c)^{2} \xi_{c}\right\}\left(\int_{0}^{1} u_{r i} u_{r j} H_{1} d \bar{x}\right)-\left\{(L / c)^{2} \xi_{r}\right\}\left(\int_{0}^{1} u_{r i} u_{r j} H_{2} d \bar{x}\right) \\
& -\left\{\left(\phi_{t} / 24\right)(c / L)^{2}\left(\xi_{c} / \phi_{c}\right)\right\}\left(\int_{0}^{L} u_{r i}^{\prime \prime} u_{r j}^{\prime \prime} H_{1} d \bar{x}\right)-\left\{\left(\phi_{t} / 24\right)(c / L)^{2}\left(\xi_{r} / \phi_{r}\right)\right\}\left(\int_{0}^{L} u_{r i}^{\prime \prime} u_{r j}^{\prime \prime} H_{2} d \bar{x}\right) \\
& \left(K_{34}\right)_{i j}=\left\{(L / c)^{2} \xi_{c}\right\}\left(\int_{0}^{1} u_{r i} u_{s j} H_{1} d \bar{x}\right)+\left\{(L / c)^{2} \xi_{r}\right\}\left(\int_{0}^{1} u_{r i} u_{s j} H_{2} d \bar{x}\right) \\
& +\left\{\left(\phi_{b} / 24\right)(c / L)^{2}\left(\xi_{c} / \phi_{c}\right)\right\}\left(\int_{0}^{1} u_{r i}^{\prime \prime} u_{s j}^{\prime \prime} H_{1} d \bar{x}\right)+\left\{\left(\phi_{b} / 24\right)(c / L)^{2}\left(\xi_{r} / \phi_{r}\right)\right\} \\
& \left(\int_{0}^{1} u_{r i}^{\prime \prime} u_{s j}^{\prime \prime} H_{2} d \bar{x}\right)
\end{aligned}
$$




$$
\begin{aligned}
& \left(K_{41}\right)_{i j}=\left\{\left(\xi_{c} / 2\right)(L / c)\left(1+d_{t} / c\right)\right\}\left(\int_{0}^{1} u_{s i}^{\prime} w_{m j} H_{1} d \bar{x}\right)+\left\{\left(\xi_{r} / 2\right)(L / c)\left(1+d_{t} / c\right)\right\} \\
& \left(\int_{0}^{1} u_{s i}^{\prime} w_{m j} H_{2} d \bar{x}\right) \\
& \left(K_{42}\right)_{i j}=\left\{\left(\xi_{c} / 2\right)(L / c)\left(1+d_{b} / c\right)\right\}\left(\int_{0}^{1} u_{s i}^{\prime} w_{q j} H_{1} d \bar{x}\right)+\left\{\left(\xi_{r} / 2\right)(L / c)\left(1+d_{b} / c\right)\right\} \\
& \left(\int_{0}^{1} u_{s i}^{\prime} w_{q j} H_{2} d \bar{x}\right) \\
& \left(K_{43}\right)_{i j}=\left\{(L / c)^{2} \xi_{c}\right\}\left(\int_{0}^{1} u_{s i} u_{r j} H_{1} d \bar{x}\right)+\left\{(L / c)^{2} \xi_{r}\right\}\left(\int_{0}^{1} u_{s i} u_{r j} H_{2} d \bar{x}\right) \\
& +\left\{\left(\phi_{t} / 24\right)(c / L)^{2}\left(\xi_{c} / \phi_{c}\right)\right\}\left(\int_{0}^{1} u_{s i}^{\prime \prime} u_{r j}^{\prime \prime} H_{1} d \bar{x}\right)+\left\{\left(\phi_{t} / 24\right)(c / L)^{2}\left(\xi_{r} / \phi_{r}\right)\right\} \\
& \left(\int_{0}^{1} u_{s i}^{\prime \prime} u_{r j}^{\prime \prime} H_{2} d \bar{x}\right) \\
& \left(K_{44}\right)_{i j}=\left(-\phi_{b}\right)\left(\int_{0}^{1} u_{s i}^{\prime} u_{s j}^{\prime} d \bar{x}\right)-\left\{(L / c)^{2} \xi_{c}\right\}\left(\int_{0}^{1} u_{s i} u_{s j} H_{1} d \bar{x}\right)-\left\{(L / c)^{2} \xi_{r}\right\}\left(\int_{0}^{1} u_{s i} u_{s j} H_{2} d \bar{x}\right) \\
& -\left\{\left(\phi_{b} / 24\right)(c / L)^{2}\left(\xi_{c} / \phi_{c}\right)\right\}\left(\int_{0}^{1} u_{s i}^{\prime \prime} u_{s j}^{\prime \prime} H_{1} d \bar{x}\right)-\left\{\left(\phi_{b} / 24\right)(c / L)^{2}\left(\xi_{r} / \phi_{r}\right)\right\} \\
& \left(\int_{0}^{1} u_{s i}^{\prime \prime} u_{s j}^{\prime \prime} H_{2} d \bar{x}\right) \\
& {\left[F_{11}\right]_{i j}=\int_{0}^{1} w_{q i}^{\prime} w_{q j}^{\prime} d \bar{x}, \quad\left[F_{22}\right]_{i j}=\int_{0}^{1} w_{m i}^{\prime} w_{m j}^{\prime} d \bar{x}}
\end{aligned}
$$

Here $t$ is for top layer and $b$ is for bottom layer. In the above relations, $w_{() i}^{\prime}=\partial w_{() i} / \partial \bar{x}$, and the sub matrices, which are not covered by the above elements, should be treated as null.

\section{Appendix B: Shape functions used for finding the numerical results}

For Simply supported beam:

$$
w_{m}(\bar{x})=\sin (m \pi \bar{x}), \quad w_{q}(\bar{x})=\sin (\bar{q} \pi \bar{x}),
$$


$u_{r}(\bar{x})=\cos (\bar{r} \pi \bar{x})$ And $u_{s}(\bar{x})=\cos (\bar{s} \pi \bar{x})$ where $m=1,2, \ldots N, \bar{q}=(q-N), \bar{r}=(r-2 N)$ and $\bar{s}=(s-3 N)$

For clamped-guided, clamped-free end conditions the following approximate functions are admissible functions satisfying as many as boundary conditions (Ray and Kar [20]).

For clamped-guided beam, the approximate functions can be as follows.

$$
\begin{aligned}
w_{m}(\bar{x})= & (m+3)(m+2)(m+1)\left\{2+\left(2-\mu_{1}\right) m\right\} \bar{x}^{(m+1)}-\left[2(m+3)(m+1)^{2}\left\{1+\left(2-\mu_{1}\right) m\right\}\right. \\
& +\mu_{1}\left\{(m+1) /\left\{2(m+2)+\left(2-\mu_{1}\right)(m+2) m\right] \bar{x}^{(m+2)}\right. \\
& +\left[(m+2)(m+1)^{2} m\left(2-\mu_{1}\right)-\mu_{1} m(m+1) /\left\{2(m+3)+\left(2-\mu_{1}\right)(m+3) m\right\}\right] \bar{x}^{(m+3)} \\
w_{q}(\bar{x})= & (\bar{q}+3)(\bar{q}+2)(\bar{q}+1)\left\{2+\left(2-\mu_{2}\right) \bar{q}\right\} \bar{x}^{(\bar{q}+1)}-\left[2(\bar{q}+3)(\bar{q}+1)^{2}\left\{1+\left(2-\mu_{2}\right) \bar{q}\right\}\right. \\
& +\mu_{2}\left\{(\bar{q}+1) /\left\{2(\bar{q}+2)+\left(2-\mu_{2}\right)(\bar{q}+2) \bar{q}\right] \bar{x}^{(\bar{q}+2)}\right. \\
& +\left[(\bar{q}+2)(\bar{q}+1)^{2} q\left(2-\mu_{2}\right)-\mu_{2} \bar{q}(\bar{q}+1) /\left\{2(\bar{q}+3)+\left(2-\mu_{2}\right)(\bar{q}+3) q\right\}\right] \bar{x}^{(\bar{q}+3)}
\end{aligned}
$$

where $\mu_{1}=Y /(1+Y)$ and $Y=3\left(1+(c / d t)^{2}\right), \mu_{2}=X /(1+X)$ and $X=3\left(1+(c / d b)^{2}\right)$

$$
\begin{aligned}
& u_{r}(\bar{x})=(\bar{r}+1) \bar{x}^{r}-\left[2(\bar{r}+3)(\bar{r}+2)(\bar{r}+1)+\bar{r}\left\{1+\mu_{1} /\left(2+2 \bar{r}-\mu_{1} \bar{r}\right)\right\}\right] \bar{x}^{(\bar{r}+1)} \\
& u_{\bar{s}}(\bar{x})=(s+1) \bar{x}^{\bar{s}}-\left[2(\bar{s}+3)(\bar{s}+2)(\bar{s}+1)+\bar{s}\left\{1+\mu_{1} /\left(2+2 s-\mu_{1} \bar{s}\right)\right\}\right] \bar{x}^{(\bar{s}+1)}
\end{aligned}
$$

and $m=1,2, \ldots N, \bar{q}=(q-N), \bar{r}=(r-2 N)$ and $\bar{s}=(s-3 N)$.

For clamped-free beam, the shape functions can be as follows

$$
\begin{aligned}
w_{m}(\bar{x})= & (m+3)(m+2)\left\{(m+2)(m+1)-\mu_{2}\right\} \bar{x}^{(m+1)}+\left[2(m+3)(m+1)\left\{\mu_{2}-m(m+2)\right\}\right. \\
& \left.\mu_{1} m(m+1) /\left\{(m+2)(m+1)-\mu_{2}\right\}\right]+\left[(m+2)(m+1)\left\{-\mu_{2}+m(m+1)\right\}-\mu_{1} m(m+1)^{2}\right. \\
& /\left\{(m+3)(m+2)(m+1)-(m+3) \mu_{2}\right\} \bar{x}^{(m+3)} \\
w_{q}(\bar{x})= & (\bar{q}+3)(\bar{q}+2)\left\{(\bar{q}+2)(\bar{q}+1)-\mu_{2}\right\} \bar{x}^{(\bar{q}+1)}+\left[2(\bar{q}+3)(\bar{q}+1)\left\{\mu_{2}-\bar{q}(\bar{q}+2)\right\}\right. \\
& \left.\mu_{1} \bar{q}(\bar{q}+1) /\left\{(\bar{q}+2)(\bar{q}+1)-\mu_{2}\right\}\right]+\left[(\bar{q}+2)(\bar{q}+1)\left\{-\mu_{2}+\bar{q}(\bar{q}+1)\right\}-\mu_{1} \bar{q}(\bar{q}+1)^{2}\right. \\
& /\left\{(\bar{q}+3)(\bar{q}+2)(\bar{q}+1)-(\bar{q}+3) \mu_{2}\right\} \bar{x}^{(\bar{q}+3)} \\
u_{r}(\bar{x})= & (\bar{r}+1) x^{\bar{r}}-\bar{r} \bar{x}^{(\bar{r}+1)} \quad u_{s}(\bar{x})=(\bar{s}+1) x^{\bar{r}}-\bar{s} \bar{x}^{(\bar{s}+1)}
\end{aligned}
$$

where $m=1,2, \ldots . N, \bar{q}=(q-N), \bar{r}=(r-2 N)$ and $\bar{s}=(s-3 N)$.

\section{References}

[1] Y. Frostig and M. Baruch, Bending of sandwich beams with transversely flexible core, American Institute of Aeronautics and Astronautics Journal 27 (1990), 523-531.

[2] Y. Frostig, Behavior of delaminated sandwich beam with transversely flexible core, High order theory, Composite Structures 20 (1992), 1-16.

[3] E. Bozhevolnaya and Y. Frostig, Nonlinear closed-form high-order analysis of curved sandwich panels, Composite Structures 38 (1994), 383-394.

[4] Y. Frostig, On stress concentration in the bending of sandwich beams with transversely flexible core, Composite Structures 24 (1993), $161-169$.

[5] Y. Frostig and M. Baruch, Free vibrations of sandwich beam with transversely flexible core: higher order approach, Journal of Sound and Vibration 176 (1994), 195-208.

[6] L. Librescu and T. Hause, Recent developments in the modeling and behaviour of advanced sandwich constructions, Composite Structures 48 (2000), 1-17.

[7] T. Shiga, A. Okada and T. Kurauchi, Magneto viscoelastic behavior of composite gels, Journal of Applied Polymer Science 58 (1995), 787-792.

[8] M.R. Jolly, J.D. Carlson, B.C. Munoz and A. Bullions, The magneto viscoelastic response of elastomer composites consisting of ferrous particles embedded in a polymer matrix, Journal of Intelligent Material Systems and Structures 7 (1996), 613-622.

[9] J.D. Carlson and M.R. Jolly, MR Fluid, foam and elastomer devices, Mechatronics 10 (2000), 555-569. 
[10] Y. Zhu, Effects of structures on rheology in a model magnetorheological fluid, International Journal of Modern Physics B 13 (1999), 2044-2051.

[11] O. Volkava, Flow-induced structures in magnetorheological suspensions, Journal of Magnetism and Magnetic Materials 201 (1999), 66-69.

[12] G.Y. Zhou and P.Q. Zhang, Investigation of the dynamic mechanical behavior of double-barreled configuration in MRF damper, Smart Materials and Structures 11 (2002), 230-238.

[13] J.M. Ginder, M.E. Nichols, L.D. Elie and J.L. Tardiff, Magnetorheological Elastomers: Properties and Applications, SPIE Conference on Smart Materials Technologies (Bellingham, W.A: SPIE Optical Engineering Press), 1999, 131-138.

[14] S.A. Demchuk and V.A. Kuzmin, Viscoelastic properties of magnetorheological elastomer in the regime of dynamic deformation, Journal of Engineering Physics and Thermophysics 75 (2002), 396-400.

[15] G.Y. Zhou and Q. Wang, Magnetorheological elastomer-based smart sandwich beam with nonconductive skins, Smart Materials and Structures 14 (2005), 1001-1009.

[16] G.Y. Zhou and Q. Wang, Use of magnetorheological elastomer in adaptive sandwich beam with conductive skins. Part I: Magnetoelastic loads in conductive skins, International Journal of Solids and Structures 43 (2006), 5386-5402.

[17] G.V. Stepanov, S.S. Abramchuk, D.A. Grishin, L.V. Nikitin and A.R Kramarenko, Effect of a homogeneous magnetic field on the viscoelastic behavior of magnetic elastomers, Polymer Testing 48 (2007), 488-495.

[18] A.H. Nayfeh and D.T. Mook, Non-linear Oscillations, Wiley Interscience, New York, 1979.

[19] M.P. Cartmell, Introduction to Linear and Non-linear parametric Vibrations, Chapman and Hall, London, 1990.

[20] K. Ray and R.C. Kar, Parametric instability of a sandwich beam with various boundary conditions, Computers and Structures 55 (1995), $857-870$.

[21] K. Ray and R.C. Kar, Parametric instability of multi-layered sandwich beams, Journal of Sound and Vibration 193 (1996), 631-644.

[22] K. Ray and R.C. Kar, Parametric instability of a dual-cored sandwich beam, Computers and Structures 61 (1996), 665-671.

[23] S. K. Dwivedy, K.C. Sahu and S.K. Babu, Parametric instability regions of three layered soft-core sandwich beam using higher order theory, Journal of Sound and Vibration 304 (2007), 326-344.

[24] S. K. Dwivedy and M. Srinivas, Parametric instability regions of a soft cored sandwich beam with magnetorheological elastomer and non-conductive skins, Proceedings of the 52nd Congress of the Indian Society of Theoretical and Applied Mechanics, December 2008, pp. 117-123.

[25] www.alulight.com (for the details of alulight ${ }^{\circledR}$ properties). 

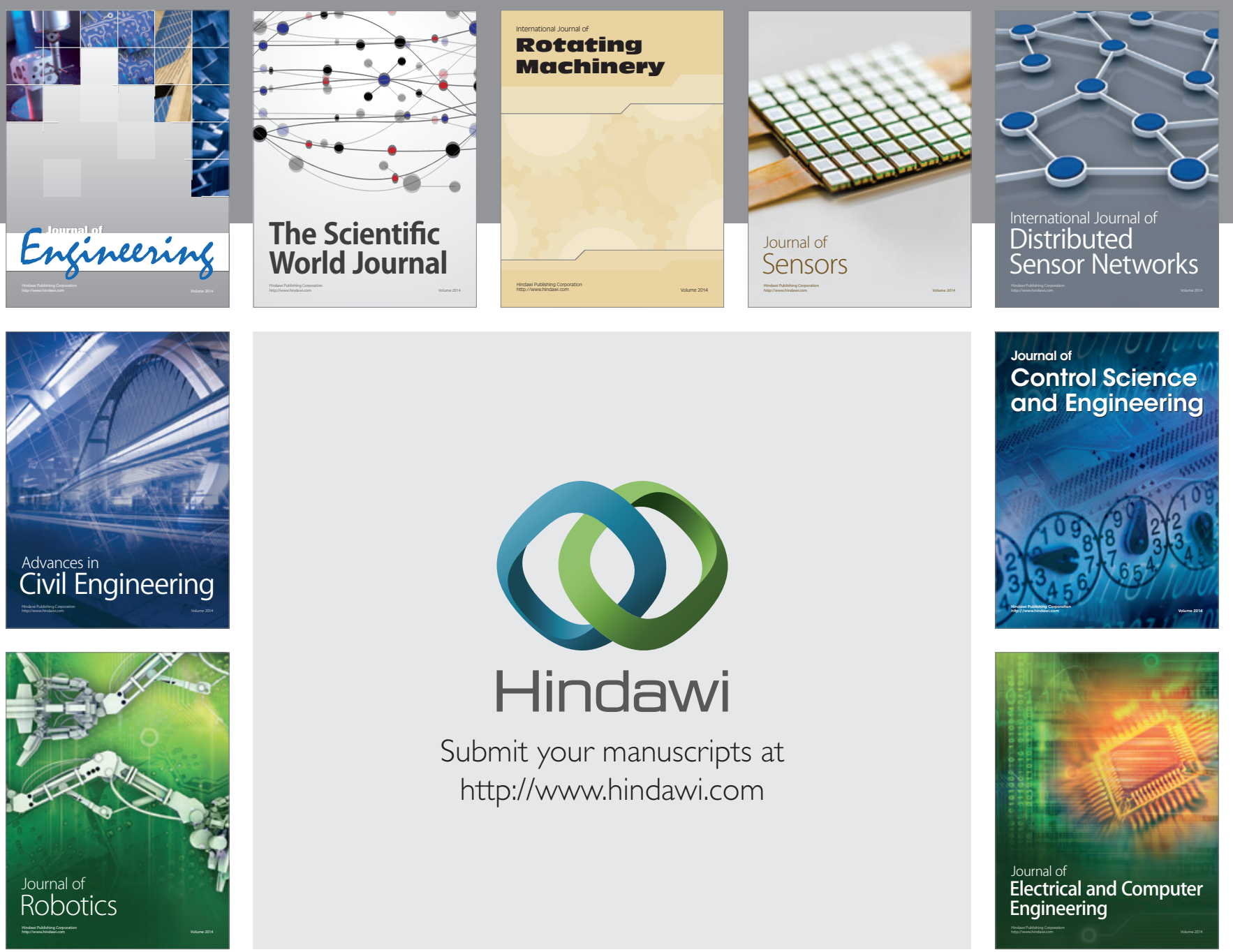

Submit your manuscripts at

http://www.hindawi.com
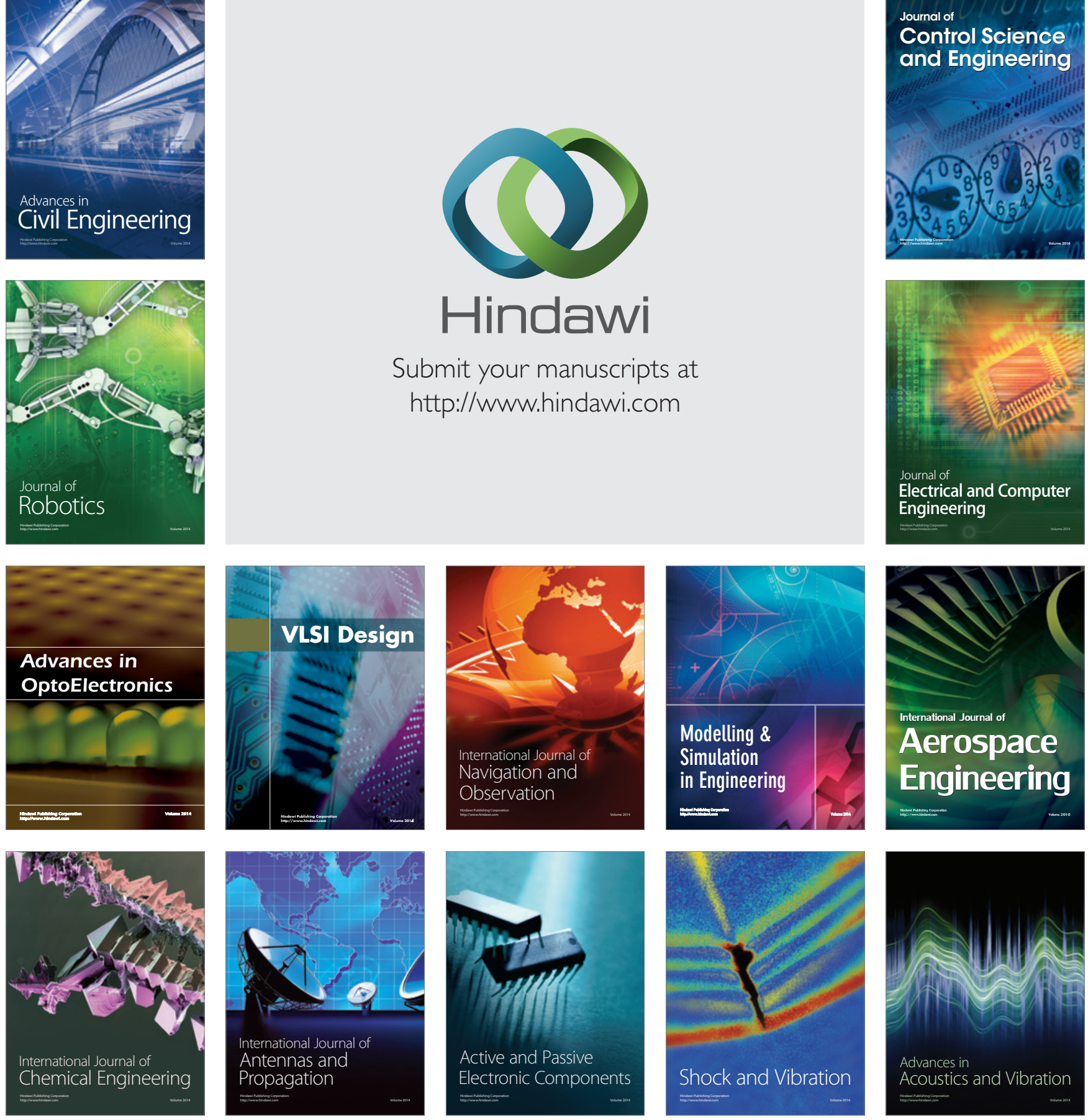